\title{
NONDISPERSIVE VANISHING AND BLOW UP AT INFINITY FOR THE ENERGY CRITICAL NONLINEAR SCHRÖDINGER EQUATION IN $\mathbb{R}^{3}$
}

\author{
C. ORTOLEVA AND G. PERELMAN
}

Dedicated to the memory of Vladimir Savelievich Buslaev

\begin{abstract}
The following energy critical focusing nonlinear Schrödinger equation in $\mathbb{R}^{3}$ is considered: $i \psi_{t}=-\Delta \psi-|\psi|^{4} \psi$; it is proved that, for any $\nu$ and $\alpha_{0}$ sufficiently small, there exist radial finite energy solutions of the form $\psi(x, t)=$ $e^{i \alpha(t)} \lambda^{1 / 2}(t) W(\lambda(t) x)+e^{i \Delta t} \zeta^{*}+o_{\dot{H}^{1}}(1)$ as $t \rightarrow+\infty$, where $\alpha(t)=\alpha_{0} \ln t, \lambda(t)=t^{\nu}$, $W(x)=\left(1+\frac{1}{3}|x|^{2}\right)^{-1 / 2}$ is the ground state, and $\zeta^{*}$ is arbitrary small in $\dot{H}^{1}$.
\end{abstract}

\section{$\S 1$. INTRODUCTION}

1.1. Setting of the problem and statement of the result. In this paper we consider the energy critical focusing nonlinear Schrödinger equation

$$
\begin{aligned}
i \psi_{t} & =-\Delta \psi-|\psi|^{4} \psi, \quad x \in \mathbb{R}^{3}, \\
\left.\psi\right|_{t=0} & =\psi_{0} \in \dot{H}^{1}\left(\mathbb{R}^{3}\right) .
\end{aligned}
$$

The Cauchy problem (1.1) is locally well posed and the solutions during their life span satisfy conservation of energy:

$$
E(\psi(t)) \equiv \int\left(|\nabla \psi(x, t)|^{2}-\frac{1}{3}|\psi(x, t)|^{6}\right) d x=E\left(\psi_{0}\right) .
$$

The problem is energy critical in the sense that both (1.1) and (1.2) are invariant with respect to the scaling $\psi(x, t) \rightarrow \lambda^{1 / 2} \psi\left(\lambda x, \lambda^{2} t\right), \lambda \in \mathbb{R}_{+}$. For $\dot{H}^{1}$ small data, one has global existence and scattering. In the case of large data, blow up may occur. Indeed, the classical virial identity

$$
\frac{d^{2}}{d t^{2}} \int|x|^{2}|\psi(x, t)|^{2} d x=8 \int\left(|\nabla \psi(x, t)|^{2}-|\psi(x, t)|^{6}\right) d x
$$

shows that if $x \psi_{0} \in L^{2}\left(\mathbb{R}^{3}\right)$ and $E\left(\psi_{0}\right)<0$, then the solution breaks down in finite time.

Furthermore, equation (1.1) admits an explicit stationary solution (ground state):

$$
W(x)=\left(1+\frac{1}{3}|x|^{2}\right)^{-1 / 2}, \quad \Delta W+W^{5}=0,
$$

so that scattering cannot always occur even for solutions that exist globally in time.

The ground state $W$ plays an important role in the dynamics of (1.1). It was proved by Kenig and Merle [6] that $E(W)$ is an energy threshold for the dynamics in the following sense. If $\psi_{0}$ is radial and $E\left(\psi_{0}\right)<E(W)$, then:

2010 Mathematics Subject Classification. Primary 35Q55.

Key words and phrases. Energy critical focusing nonlinear Schrödinger equation, Cauchy problem, ground state, blow up. 
(i) the solution of (1.1) is global and scatters to zero as a free wave in both directions, provided $\left\|\nabla \psi_{0}\right\|_{L^{2}}<\|\nabla W\|_{L^{2}}$;

(ii) the solution blows up in finite time in both directions, provided $\psi_{0} \in L^{2}$ and $\left\|\nabla \psi_{0}\right\|_{L^{2}}>\|\nabla W\|_{L^{2}}$.

The behavior of radial solutions with critical energy $E\left(\psi_{0}\right)=E(W)$ was classified by Duyckaerts and Merle [5. In this case, in addition to the finite time blow up and scattering to zero (and $W$ itself), there exist solutions that converge as $t \rightarrow \infty$ to a rescaled ground state. In the case of energy slightly greater than $E(W)$, the dynamics is expected to be more rich and to include solutions that behave as $t \rightarrow \infty$ like $e^{i \alpha(t)} \lambda^{1 / 2}(t) W(\lambda(t) x)$ with fairly general $\alpha(t)$ and $\lambda(t)$. For a closely related model of the critical wave equation, the existence of solutions of this type with $\lambda(t) \rightarrow \infty$ (blow up at infinity) and $\lambda(t) \rightarrow 0, t \lambda(t) \rightarrow \infty$ (nondispersive vanishing) was recently proved by Donninger and Krieger [4. Our objective in this paper is to obtain a similar result for NLS (1.1). More precisely, we prove the following.

Theorem 1.1. There exists $\beta_{0}>0$ such that for any $\nu, \alpha_{0} \in \mathbb{R}$ with $|\nu|+\left|\alpha_{0}\right| \leq \beta_{0}$ and any $\delta>0$, there exists $T>0$ and a radial solution $\psi \in C\left([T,+\infty), \dot{H}^{1} \cap \dot{H}^{2}\right)$ of (1.1) of the form

$$
\psi(x, t)=e^{i \alpha(t)} \lambda^{1 / 2}(t) W(\lambda(t) x)+\zeta(x, t),
$$

where $\lambda(t)=t^{\nu}, \alpha(t)=\alpha_{0} \ln t$, and $\zeta(t)$ satisfies:

$$
\begin{aligned}
& \|\zeta(t)\|_{\dot{H}^{1} \cap \dot{H}^{2}} \leq \delta \\
& \|\zeta(t)\|_{L^{\infty}} \leq C t^{-\frac{1+\nu}{2}}, \\
& \left\|\langle\lambda(t) x\rangle^{-1} \zeta(t)\right\|_{L^{\infty}} \leq C t^{-1-\frac{3}{2} \nu}
\end{aligned}
$$

for all $t \geq T$. The constants $C$ here and below are independent of $\nu, \alpha_{0}$, and $\delta$. Furthermore, there exists $\zeta^{*} \in \dot{H}^{s}$, for any $s>\frac{1}{2}-\nu$, such that, as $t \rightarrow+\infty, \zeta(t)-e^{i t \Delta} \zeta^{*} \rightarrow 0$ in $\dot{H}^{1} \cap \dot{H}^{2}$.

Remark 1.2. In fact, Theorem 1.1 remains valid with $\dot{H}^{2}$ replaced by $\dot{H}^{k}$ for any $k \geq 2$ (with $\beta_{0}$ depending on $k$ ).

Remark 1.3. The restriction on $\nu$ and $\alpha_{0}$ that appears in Theorem 1.1 seems to be technical. One might expect the same result to be true for any $\nu>-1 / 2$ and any $\alpha_{0} \in \mathbb{R}$.

Remark 1.4. The solutions we construct to prove the theorem belong, in fact, to $\dot{H}^{\frac{1}{2}-\nu+}$.

Remark 1.5. Using the techniques developed in this paper one can prove the existence of radial finite time blow up solutions of the form

$$
\begin{aligned}
\psi(x, t) & =e^{i \alpha(t)} \lambda^{1 / 2}(t) W(\lambda(t) x)+\zeta(x, t), \\
\lambda(t) & =(T-t)^{-1 / 2-\nu}, \quad \alpha(t)=\alpha_{0} \ln (T-t),
\end{aligned}
$$

where $\zeta(t)$ is arbitrarily small in $\dot{H}^{1} \cap \dot{H}^{2}$, and $\nu>1$, and $\alpha_{0} \in \mathbb{R}$ can be chosen arbitrarily. For the critical wave equation an analogous result was proved by Krieger, Schlag, Tataru in [8], see also 9] for a similar construction in the context of the critical Schrödinger map equation.

1.2. Outline of the paper. The paper is organized as follows. In $\S 2$, we construct (Proposition 2.1) a sufficiently good approximate solution of (1.1) very much in the spirit of [4, 8, 9]. In $\S 3$, we build up an exact solution by solving the problem for the small remainder with zero initial data at infinity, the main technical tool of the construction 
being some suitable energy type estimates for the linearized evolution. These estimates are proved in $\S 4$.

\section{§2. Approximate solutions}

In this section we prove the following result.

Proposition 2.1. For any $\nu$ and $\alpha_{0}$ sufficiently small and any $0<\delta \leq 1$, there exists a radial approximate solution $\psi^{a p} \in C^{\infty}\left(\mathbb{R}^{3} \times \mathbb{R}_{+}^{*}\right)$ of (1.1) such that the following holds true for $t \geq T$ with some $T=T\left(\nu, \alpha_{0}, \delta\right)>0$.

(i) $\psi^{a p}$ has the form $\psi^{a p}(x, t)=e^{i \alpha(t)} \lambda^{1 / 2}(t)\left(W(\lambda(t) x)+\chi^{a p}(\lambda(t) x, t)\right)$, where the function $\chi^{a p}(y, t)$ with $y=\lambda(t) x$ satisfies

$$
\begin{aligned}
& \left\|\chi^{a p}(t)\right\|_{\dot{H}^{k}} \leq C \delta^{\nu+k-1 / 2} t^{-\nu(k-1)}, \quad k=1,2, \\
& \left\|\chi^{a p}(t)\right\|_{L^{\infty}} \leq C t^{-(1+2 \nu) / 2}, \\
& \left\||y|^{-1} \chi^{a p}(t)\right\|_{L^{\infty}}+\left\|\nabla \chi^{a p}(t)\right\|_{L^{\infty}} \leq C t^{-1-2 \nu}, \\
& \left\||y|^{-2} \chi^{a p}(t)\right\|_{L^{\infty}}+\left\||y|^{-1} \nabla_{y} \chi^{a p}(t)\right\|_{L^{\infty}} \leq C\left(|\nu|+\left|\alpha_{0}\right|\right) t^{-1-2 \nu}, \\
& \left\|\nabla^{2} \chi^{a p}(t)\right\|_{L^{\infty}} \leq C\left(|\nu|+\left|\alpha_{0}\right|\right) t^{-1-2 \nu} .
\end{aligned}
$$

Furthermore, there exists $\zeta^{*} \in \dot{H}^{s}$, for any $s>\frac{1}{2}-\nu$, such that, as $t \rightarrow+\infty$,

$$
e^{i \alpha(t)} \lambda^{1 / 2}(t) \chi^{a p}(\lambda(t) \cdot, t)-e^{i t \Delta} \zeta^{*} \rightarrow 0
$$

in $\dot{H}^{1} \cap \dot{H}^{2}$.

(ii) The corresponding error $R=-i \psi_{t}^{a p}-\Delta \psi^{a p}-\left|\psi^{a p}\right|^{4} \psi^{a p}$ satisfies

$$
\|R(t)\|_{\dot{H}^{k}} \leq t^{-\left(2+\frac{1}{8}\right)(1+2 \nu)+\nu(k+1)}, \quad k=0,1,2 .
$$

The construction of $\psi^{a p}(t)$ will be achieved by considering separately the three regions that correspond to three different space scales: the inner region with the scale $t^{\nu}|x| \lesssim 1$, the self-similar region where $|x|=O\left(t^{1 / 2}\right)$, and finally, the remote region where $|x|=$ $O(t)$. In the inner region the solution will be constructed as a perturbation of the profile $e^{i \alpha_{0} \ln t} t^{\nu / 2} W\left(t^{\nu} x\right)$. The self-similar and remote regions are regions where the solution is small and is described essentially by the linear equation $i \psi_{t}=-\Delta \psi$. In the selfsimilar region, the profile of the solution will be determined uniquely by the matching conditions coming out from the inner region, while in the remote region the profile remains essentially a free parameter of the construction, only the limiting behavior at the origin being prescribed by the matching procedure.

2.1. The inner region. We start by treating the inner region $0 \leq t^{\nu}|x| \leq 10 t^{1 / 2+\nu-\epsilon_{1}}$, with $0<\epsilon_{1}<1 / 2+\nu$ to be fixed later. Writing $\psi(x, t)$ as $\psi(x, t)=e^{i \alpha(t)} \lambda^{1 / 2}(t) u(\rho, t)$, $\rho=\lambda(t)|x|$, from (1.1) we get

$$
i t^{-2 \nu} u_{t}-\alpha_{0} t^{-(1+2 \nu)} u+i \nu t^{-(1+2 \nu)}\left(\frac{1}{2}+\rho \partial_{\rho}\right) u=-\triangle u-|u|^{4} u .
$$

Write $u(\rho, t)=W(\rho)+\chi(\rho, t)$. Then $\vec{\chi}(t)=\left(\begin{array}{l}\chi(t) \\ \bar{\chi}(t)\end{array}\right)$ solves

$$
i t^{-2 \nu} \vec{\chi}_{t}=H \vec{\chi}+\mathcal{N}(\chi)
$$


where

$$
\begin{aligned}
H & =-\triangle \sigma_{3}-3 W^{4} \sigma_{3}-2 W^{4} \sigma_{3} \sigma_{1}, \quad \sigma_{1}=\left(\begin{array}{ll}
0 & 1 \\
1 & 0
\end{array}\right), \quad \sigma_{3}=\left(\begin{array}{cc}
1 & 0 \\
0 & -1
\end{array}\right), \\
\mathcal{N}(\chi) & =\left(\begin{array}{c}
N(\chi) \\
-N(\chi)
\end{array}\right), \quad N(\chi)=N_{0}+N_{1}(\chi)+N_{2}(\chi), \\
N_{0} & =\alpha_{0} t^{-(1+2 \nu)} W-i \nu t^{-(1+2 \nu)} W_{1}, \quad W_{1}(\rho)=\left(\frac{1}{2}+\rho \partial_{\rho}\right) W(\rho) \\
N_{1}(\chi) & =\alpha_{0} t^{-(1+2 \nu)} \chi-i \nu t^{-(1+2 \nu)}\left(\frac{1}{2}+\rho \partial_{\rho}\right) \chi \\
N_{2}(\chi) & =-|W+\chi|^{4}(W+\chi)+W^{5}+3 W^{4} \chi+2 W^{4} \bar{\chi} .
\end{aligned}
$$

We look for a solution of (2.8) of the form

$$
\chi(\rho, t)=\sum_{k=1}^{\infty} t^{-k(1+2 \nu)} \chi_{k}(\rho) .
$$

Substituting (2.9) in (2.8) and identifying the terms with the same powers of $t$, we get the following system for $\left\{\chi_{k}\right\}_{k \geq 1}$ :

$$
H \vec{\chi}_{k}=\mathcal{D}_{k}, \quad k \geq 1,
$$

where $\mathcal{D}_{k}=\left(\begin{array}{c}D_{k} \\ -\overline{D_{k}}\end{array}\right)$,

$$
\begin{aligned}
& D_{1}=-\alpha_{0} W+i \nu W_{1}, \\
& D_{k}=D_{k}^{(1)}+D_{k}^{(2)}, \quad k \geq 2,
\end{aligned}
$$

$D_{k}^{(1)}$ and $D_{k}^{(2)}$ being the contributions of $i t^{-2 \nu} \chi_{t}-N_{1}(\chi)$ and $-N_{2}(\chi)$, respectively:

$$
\begin{aligned}
D_{k}^{(1)} & =-i(1+2 \nu)(k-1) \chi_{k-1}-\alpha_{0} \nu \chi_{k-1}+i \nu\left(\frac{1}{2}+\rho \partial_{\rho}\right) \chi_{k-1}, \\
N_{2}(\chi) & =-\sum_{k=2}^{\infty} t^{-k(1+2 \nu)} D_{k}^{(2)}(\rho) .
\end{aligned}
$$

Note that $D_{k}$ depends on $\chi_{p}, 1 \leq p \leq k-1$ only:

$$
D_{k}=D_{k}\left(\rho ; \chi_{p}, 1 \leq p \leq k-1\right) .
$$

We subject (2.10) to zero initial conditions at $0: \chi_{k}(0)=\partial_{\rho} \chi_{k}(0)=0$.

Lemma 2.2. System (2.10) has a unique solution $\left\{\chi_{k}\right\}_{k \geq 1}$ such that:

i) for any $k \geq 1, \chi_{k}$ is a $C^{\infty}$-function with an even Taylor expansion at $\rho=0$ that starts at order $2 k$;

ii) as $\rho \rightarrow+\infty$, the function $\chi_{k}, k \geq 1$, has an asymptotic expansion of the form

$$
\chi_{k}(\rho)=\sum_{l=0}^{k} \sum_{j \leq 2 k-2 l-1} \alpha_{l, j}^{(k)}(\ln \rho)^{l} \rho^{j},
$$

with some coefficients $\alpha_{l, j}^{(k)}$ verifying $\alpha_{k, 2 m}^{(k)}=0$ for all $k, m$. The asymptotic expansion (2.11) can be differentiated any number of times with respect to $\rho$.

Proof. It will be convenient for us to rewrite (2.10) as

$$
L_{+} v_{k}^{+}=G_{k}^{+}, \quad L_{-} v_{k}^{-}=G_{k}^{-}, \quad k \geq 1,
$$


where

$$
\begin{aligned}
v_{k}^{+} & =\operatorname{Re} \chi_{k}, & & v_{k}^{-}=\operatorname{Im} \chi_{k}, \\
G_{k}^{+} & =\operatorname{Re} D_{k}, & & G_{k}^{-}=\operatorname{Im} D_{k}, \\
L_{+} & =-\triangle-5 W^{4}, & & L_{-}=-\triangle-W^{4} .
\end{aligned}
$$

For $k=1$, (2.12) gives

$$
L_{+} v_{1}^{+}=-\alpha_{0} W, \quad L_{-} v_{1}^{-}=\nu W_{1} .
$$

The homogeneous equation $L_{ \pm} f=0$ has two explicit solutions $\Phi_{ \pm}, \Theta_{ \pm}$given by

$$
\begin{aligned}
& \Phi_{-}(\rho)=W(\rho), \quad \Theta_{-}(\rho)=\left(1+\frac{\rho^{2}}{3}\right)^{-1 / 2}\left(\frac{\rho}{3}-\frac{1}{\rho}\right), \\
& \Phi_{+}(\rho)=W_{1}(\rho), \quad \Theta_{+}(\rho)=-2\left(1+\frac{\rho^{2}}{3}\right)^{-3 / 2}\left(\frac{1}{\rho}-2 \rho+\frac{\rho^{3}}{9}\right) .
\end{aligned}
$$

Therefore, solving (2.13) with zero initial conditions at the origin, we obtain

$$
\begin{aligned}
& v_{1}^{+}(\rho)=\alpha_{0} \int_{0}^{\rho} s^{2}\left(\Theta_{+}(\rho) \Phi_{+}(s)-\Theta_{+}(s) \Phi_{+}(\rho)\right) W(s) d s \\
& v_{1}^{-}(\rho)=-\nu \int_{0}^{\rho} s^{2}\left(\Theta_{-}(\rho) \Phi_{-}(s)-\Theta_{-}(s) \Phi_{-}(\rho)\right) W_{1}(s) d s .
\end{aligned}
$$

Since $W, W_{1}$ are even $C^{\infty}$-functions, $v_{1}^{+}$and $v_{1}^{-}$are also $C^{\infty}$-functions with even Taylor expansion at $\rho=0$ that starts at order 2. Furthermore, the asymptotic expansions of $v_{1}^{+}$ and $v_{1}^{-}$as $\rho \rightarrow+\infty$ can be obtained directly from (2.15). As claimed, we have

$$
v_{1}^{+}(\rho)+i v_{1}^{-}(\rho)=\sum_{j \leq 1} \alpha_{0, j}^{(1)} \rho^{j}+\sum_{j \leq 0} \alpha_{1, j}^{(1)} \rho^{2 j-1} \ln \rho \quad \text { as } \rho \rightarrow+\infty .
$$

Next we proceed by induction. Consider $k>1$ and assume that we have found $\chi_{i}$, $i=1, \ldots, k-1$, that satisfy i), ii). Then we can check easily that $D_{k}$ is an even $C^{\infty}$ function with a Taylor series at 0 starting at order $2(k-1)$, and that, as $\rho \rightarrow+\infty, D_{k}$ admits an asymptotic expansion of the form

$$
D_{k}(\rho)=\sum_{l=0}^{k-1} \sum_{j \leq 2 k-2 l-3} d_{j, l}^{(k)}(\ln \rho)^{l} \rho^{j}+(\ln \rho)^{k} \sum_{j \leq-5} d_{j, k}^{(k)} \rho^{j},
$$

where $d_{-2, k-1}^{(k)}=0$ and $d_{2 m, k}^{(k)}=0$, for all $m$. Therefore, solving $L_{ \pm} v_{k}^{ \pm}=G_{k}^{ \pm}$with zero conditions at $\rho=0$ we get a $C^{\infty}$ even solution $v_{k}^{ \pm}$which is $O\left(\rho^{2 k}\right)$ at the origin. Finally, the asymptotic expansion at infinity follows directly from the representation

$$
v_{k}^{ \pm}(\rho)=-\int_{0}^{\rho} s^{2}\left(\Theta_{ \pm}(\rho) \Phi_{ \pm}(s)-\Theta_{ \pm}(s) \Phi_{ \pm}(\rho)\right) G_{k}^{ \pm}(s) d s .
$$

Remark 2.3. Clearly, for any $k$, the function $\chi_{k}$ is a polynomial with respect to $\alpha_{0}$ and $\nu$ of the form

$$
\chi_{k}=\sum_{1 \leq m+n \leq k} \alpha_{0}^{m} \nu^{n} \chi_{m, n}^{k}(\rho)
$$

where the coefficients $\chi_{m, n}^{k}$ are $C^{\infty}$-functions of $\rho$ with an even Taylor expansion at 0 that starts at order $2 k$. As $\rho \rightarrow+\infty, \chi_{m, n}^{k}$ admits an asymptotic expansion of the form (2.11). 
For any $N \geq 2$, define

$$
\chi^{(N)}(\rho, t)=\sum_{k=1}^{N} t^{-k(1+2 \nu)} \chi_{k}(\rho) .
$$

From our construction it follows that $\chi^{(N)}$ verifies

$$
\left|\rho^{-k} \partial_{\rho}^{l}\left(-i t^{-2 \nu} \vec{\chi}_{t}^{(N)}+H \vec{\chi}^{(N)}+\mathcal{N}\left(\chi^{(N)}\right)\right)\right| \leq C_{N, l, k} t^{-(N+1)(1+2 \nu)}\langle\rho\rangle^{2 N-1-l-k},
$$

for any $k, l \in \mathbb{N}$, with $k+l \leq 2 N, 0 \leq \rho \leq 10 t^{\frac{1}{2}+\nu-\epsilon_{1}}, t \geq 1$.

Fix $N=27, \epsilon_{1}=\frac{1+2 \nu}{27}, 1$ and set

$$
\begin{aligned}
u_{i n}^{a p} & =W+\chi_{i n}^{a p}, \quad \chi_{i n}^{a p}=\chi^{(27)}, \\
\mathcal{R}_{i n} & =-i t^{-2 \nu} \partial_{t} u_{i n}^{a p}-\triangle u_{i n}^{a p}+\alpha_{0} t^{-1-2 \nu} u_{i n}^{a p}-i \nu t^{-1-2 \nu}\left(\frac{1}{2}+\rho \partial_{\rho}\right) u_{i n}^{a p}-\left|u_{i n}^{a p}\right|^{4} u_{i n}^{a p} .
\end{aligned}
$$

As a direct consequence of Lemma 2.2 and estimate (2.16), we obtain the following result.

Lemma 2.4. For any $\alpha_{0} \in \mathbb{R}$ and any $\nu>-\frac{1}{2}$, there exists $T=T\left(\alpha_{0}, \nu\right)>0$ such that for $t \geq T$ the following holds true.

(i) The profile $\chi_{\text {in }}^{a p}(t)$ satisfies

$$
\begin{aligned}
& \left\|\chi_{i n}^{a p}\right\|_{L^{\infty}\left(0 \leq \rho \leq 10 t^{\left.\frac{1}{2}+\nu-\epsilon_{1}\right)}\right.} \leq C\left(|\nu|+\left|\alpha_{0}\right|\right) t^{-\frac{1}{2}-\nu}, \\
& \left\|\rho^{-k} \partial_{\rho}^{l} \chi_{i n}^{a p}\right\|_{L^{\infty}\left(0 \leq \rho \leq 10 t^{\frac{1}{2}+\nu-\epsilon_{1}}\right)} \leq C\left(|\nu|+\left|\alpha_{0}\right|\right) t^{-1-2 \nu}, \quad 1 \leq k+l \leq 2, \\
& \left\|\rho^{-k} \partial_{\rho}^{l} \chi_{i n}^{a p}\right\|_{L^{2}\left(\rho^{2} d \rho, 0 \leq \rho \leq 10 t^{\frac{1}{2}+\nu-\epsilon_{1}}\right)} \leq C\left(|\nu|+\left|\alpha_{0}\right|\right) t^{-\left(\frac{1}{2}+\nu\right)\left(k+l-\frac{1}{2}\right)}, \quad k+l \leq 2 .
\end{aligned}
$$

(ii) The error $\mathcal{R}_{\text {in }}(t)$ admits the estimate

$$
\left\|\rho^{-k} \partial_{\rho}^{l} \mathcal{R}_{i n}(t)\right\|_{L^{2}\left(\rho^{2} d \rho, 0 \leq \rho \leq 10 t^{\left.\frac{1}{2}+\nu-\epsilon_{1}\right)}\right.} \leq t^{-3(1+2 \nu) / 4-\varepsilon_{1}(2 N+1 / 2)}, \quad k+l \leq 2 .
$$

2.2. The self-similar region. We next consider the self-similar region

$$
\frac{1}{10} t^{-\varepsilon_{1}} \leq|x| t^{-1 / 2} \leq 10 t^{\varepsilon_{2}}
$$

with $0<\varepsilon_{2}<1 / 2$ to be fixed later. Write $\psi(x, t)=e^{i \alpha_{0} \ln t} t^{-1 / 4} w(y, t), y=t^{-1 / 2}|x|$. Then $w(t)$ solves

$$
i t w_{t}=\left(\mathcal{L}+\alpha_{0}\right) w-|w|^{4} w,
$$

where $\mathcal{L}=-\triangle+\frac{i}{2}\left(\frac{1}{2}+y \partial_{y}\right)$.

Note that in the limit $\rho \rightarrow+\infty, y \rightarrow 0$ at least formally we have

$$
\begin{aligned}
& t^{\nu / 2}\left(W(\rho)+\sum_{k \geq 1} t^{-k(1+2 \nu)} \chi_{k}(\rho)\right) \\
& =t^{-1 / 4} \sum_{n \geq 0} \sum_{0 \leq l \leq \frac{n}{2}} t^{-\frac{1}{4}(2 n+1)(1+2 \nu)}\left(\ln y+\left(\frac{1}{2}+\nu\right) \ln t\right)^{l} \sum_{k \geq l} \alpha_{l, 2 k-n-1}^{(k)} y^{2 k-n-1},
\end{aligned}
$$

where the $\alpha_{l, j}^{(k)}, k \neq 0$, are given by Lemma 2.2, and the $\alpha_{l, j}^{(0)}$ come from the expansion of $W(\rho)$ as $\rho \rightarrow \infty$ :

$$
W(\rho)=\sum_{j \leq 0} \alpha_{0, j}^{(0)} \rho^{j}, \quad \alpha_{0,2 m}^{(0)}=0, \text { for all } m \in \mathbb{Z} .
$$

\footnotetext{
${ }^{1}$ This choice has no specific meaning here. To produce an approximate solution with an error satisfying (2.6), it suffices to require $(2 N+3) \varepsilon_{1}>3(1+2 \nu) / 2,0<\varepsilon_{1}<\frac{1+2 \nu}{20}$, see (2.20) and (2.41), (2.42).
} 
Equation (2.22) suggests the following Ansatz for w:

$$
w(y, t)=\sum_{n \geq 0} \sum_{0 \leq l \leq \frac{n}{2}} t^{-\frac{1}{4}(2 n+1)(1+2 \nu)}\left(\ln y+\left(\frac{1}{2}+\nu\right) \ln t\right)^{l} A_{n, l}(y) .
$$

As it will become clear later, to prove Proposition 2.1 it suffices to consider only first three terms in (2.23). Therefore, we look for an approximate solution of the form

$$
\begin{aligned}
w_{s s}^{a p}(y, t)=t^{-(1+2 \nu) / 4} A_{0,0}(y) & +t^{-3(1+2 \nu) / 4} A_{1,0}(y) \\
& +t^{-5(1+2 \nu) / 4}\left(A_{2,0}(y)+\left(\ln y+\left(\frac{1}{2}+\nu\right) \ln t\right) A_{2,1}(y)\right) .
\end{aligned}
$$

Substituting this in the expression $-i t w_{t}+\left(\mathcal{L}+\alpha_{0}\right) w-|w|^{4} w$, we get

$$
\begin{aligned}
-i t \partial_{t} w_{s s}^{a p} & +\left(\mathcal{L}+\alpha_{0}\right) w_{s s}^{a p}-\left|w_{s s}^{a p}\right|^{4} w_{s s}^{a p}=t^{-(1+2 \nu) / 4} S_{0,0}(y)+t^{-3(1+2 \nu) / 4} S_{1,0}(y) \\
& +t^{-5(1+2 \nu) / 4}\left(S_{2,0}(y)+\left(\ln y+\left(\frac{1}{2}+\nu\right) \ln t\right) S_{2,1}(y)\right)+S(y, t),
\end{aligned}
$$

where

$$
\begin{aligned}
S_{n, 0}(y) & =\left(\mathcal{L}+\mu_{n}\right) A_{n, 0}(y), \quad n=0,1, \\
S_{2,1}(y) & =\left(\mathcal{L}+\mu_{2}\right) A_{2,1}(y), \\
S_{2,0}(y) & =\left(\mathcal{L}+\mu_{2}\right) A_{2,0}(y)-i \nu A_{2,1}(y)-\frac{2}{y} \partial_{y} A_{2,1}(y)-\frac{A_{2,1}(y)}{y^{2}}-\left|A_{0,0}(y)\right|^{4} A_{0,0}(y), \\
S(y, t) & =-\left|w_{s s}^{a p}(y, t)\right|^{4} w_{s s}^{a p}(y, t)+t^{-5(1+2 \nu) / 4}\left|A_{0,0}(y)\right|^{4} A_{0,0}(y) .
\end{aligned}
$$

Here $\mu_{n}=\alpha_{0}+\frac{i}{4}(2 n+1)(1+2 \nu)$.

We require that $S_{n, l}=0, n=0,1,2, l=0,1$, which means that the corresponding $A_{n, l}$ must solve

$$
\left\{\begin{array}{l}
\left(\mathcal{L}+\mu_{n}\right) A_{n, 0}=0, \quad n=0,1 \\
\left(\mathcal{L}+\mu_{2}\right) A_{2,1}=0 \\
\left(\mathcal{L}+\mu_{2}\right) A_{2,0}=i \nu A_{2,1}+\frac{2}{y} \partial_{y} A_{2,1}+\frac{A_{2,1}}{y^{2}}+\left|A_{0,0}\right|^{4} A_{0,0} .
\end{array}\right.
$$

Moreover, in order to have the matching with the inner region, the $A_{n, l}$ must satisfy

$$
A_{n, l}(y)=\sum_{k \geq l} \alpha_{l, 2 k-n-1}^{(k)} y^{2 k-n-1}, \quad y \rightarrow 0 .
$$

Lemma 2.5. There exists a unique solution of (2.25) such that, as $y \rightarrow 0$, it admits an asymptotic expansion of the form

$$
A_{n, l}(y)=\sum_{k \geq l} d_{n, k, l} y^{2 k-n-1}
$$

with $d_{0,0,0}=\alpha_{0,-1}^{(0)}, d_{1,1,0}=\alpha_{0,0}^{(1)}$ and $d_{2,1,0}=\alpha_{0,-1}^{(1)}$.

Proof. First, we note that the equation $(\mathcal{L}+\mu) f=0$ has a basis of solutions $e_{1}(y, \mu)$, $e_{2}(y, \mu)$ such that

(i) $e_{1}(y, \mu)=\frac{1}{y}+\left(\mu-\frac{i}{4}\right) \widetilde{e}_{1}(y, \mu)$, where $\widetilde{e}_{1}$ is an entire function of $y$ and $\mu$, odd with respect to $y$;

(ii) $e_{2}$ is an entire function of $y$ and $\mu$ even with respect to $y$, and $e_{2}(y, \mu)=1+O\left(y^{2}\right)$ as $y \rightarrow 0$.

The first two equations in (2.25) together with (2.27) give

$$
A_{0,0}(y)=\alpha_{0,-1}^{(0)} e_{1}\left(y, \mu_{0}\right), \quad A_{1,0}(y)=\alpha_{0,0}^{(1)} e_{2}\left(y, \mu_{1}\right) .
$$


Next we consider the remaining equations in (2.25). The equation $\left(\mathcal{L}+\mu_{2}\right) A_{2,1}(y)=0$ and (2.27) yield $A_{2,1}(y)=c_{0} e_{1}\left(y, \mu_{2}\right)$ with some constant $c_{0}$. Then, for $A_{2,0}$ we have $\left(\mathcal{L}+\mu_{2}\right) A_{2,0}=F$, where

$$
F=c_{0}\left(i \nu+\frac{2}{y} \partial_{y}+y^{-2}\right) e_{1}\left(y, \mu_{2}\right)+\left|A_{0,0}\right|^{4} A_{0,0} .
$$

As $y \rightarrow 0, F$ has an asymptotic expansion of the form

$$
F(y)=\sum_{i \geq-2} \kappa_{i} y^{2 i-1},
$$

with some coefficients $\kappa_{i}, \kappa_{-2}$ and $\kappa_{-1}+c_{0}$ being independent of $c_{0}$.

Write $A_{2,0}(y)=-\frac{\kappa_{-2}}{6 y^{3}}+\widetilde{A}_{2,0}(y)$. Then $\widetilde{A}_{2,0}$ solves

$$
\left(\mathcal{L}+\mu_{2}\right) \widetilde{A}_{2,0}=\widetilde{F},
$$

where $\widetilde{F}=F+\frac{\kappa_{-2}}{6}\left(\mathcal{L}+\mu_{2}\right) \frac{1}{y^{3}}$ has the following asymptotics as $y \rightarrow 0$ :

$$
\widetilde{F}(y)=\sum_{i \geq-1} \widetilde{\kappa}_{i} y^{2 i-1}, \quad \widetilde{\kappa}_{-1}=\widetilde{\kappa}_{-1}^{0}-c_{0},
$$

with $\widetilde{\kappa}_{-1}^{0}$ independent of $c_{0}$. Take $c_{0}=\widetilde{\kappa}_{-1}^{0}$. Then $(2.29)$ has a unique solution of the form

$$
\widetilde{A}_{2,0}(y)=\alpha_{0,-1}^{(1)} e_{1}\left(y, \mu_{2}\right)+\text { an odd } C^{\infty} \text {-function. }
$$

Remark 2.6. By uniqueness, the functions $A_{n, l}$ given by Lemma 2.5 verify the matching conditions (2.26). Note also that all $A_{n, l}$ are entire functions of $\alpha_{0}$ and $\nu$.

Next we study the behavior of $A_{n, l}$ as $y \rightarrow+\infty$. For this, notice that for any $\mu \in \mathbb{C}$, equation $(\mathcal{L}+\mu) f=0$ has a basis of solutions $f_{1}(y, \mu), f_{2}(y, \mu)$ such that $y f_{1}, y f_{2}$ are smooth functions in both variables, and as $y \rightarrow+\infty$ we have

$$
f_{1}(y, \mu)=y^{-1 / 2+2 i \mu}\left(1+O\left(y^{-2}\right)\right), \quad f_{2}(y, \mu)=e^{i \frac{y^{2}}{4}} y^{-5 / 2-2 i \mu}\left(1+O\left(y^{-2}\right)\right) .
$$

These asymptotics are uniform in $\mu$ on the compact subsets of $\mathbb{C}$ and can be differentiated any number of times with respect to $y$.

Decomposing $A_{1,0}, A_{2,0}, A_{2,1}$ in the basis $f_{1}, f_{2}$, we get

$$
\begin{aligned}
& A_{n, 0}(y)=d_{1}^{n} f_{1}\left(y, \mu_{n}\right)+d_{2}^{n} f_{2}\left(y, \mu_{n}\right), \quad n=0,1, \\
& A_{2,1}(y)=d_{1}^{2} f_{1}\left(y, \mu_{2}\right)+d_{2}^{2} f_{2}\left(y, \mu_{2}\right),
\end{aligned}
$$

with some coefficients $d_{j}^{n}, j=1,2, n=0,1,2$. As a consequence, as $y \rightarrow+\infty$, we have

$$
\begin{aligned}
& A_{0,0}(y)=d_{1}^{0} y^{2 i \alpha_{0}-1-\nu}\left(1+O\left(y^{-2}\right)\right)+d_{2}^{0} e^{i y^{2} / 4} y^{-2 i \alpha_{0}-2+\nu}\left(1+O\left(y^{-2}\right)\right), \\
& A_{1,0}(y)=d_{1}^{1} y^{2 i \alpha_{0}-2-3 \nu}\left(1+O\left(y^{-2}\right)\right)+d_{2}^{1} e^{i y^{2} / 4} y^{-2 i \alpha_{0}-1+3 \nu}\left(1+O\left(y^{-2}\right)\right), \\
& A_{2,1}(y)=d_{1}^{2} y^{2 i \alpha_{0}-3-5 \nu}\left(1+O\left(y^{-2}\right)\right)+d_{2}^{2} e^{i y^{2} / 4} y^{-2 i \alpha_{0}+5 \nu}\left(1+O\left(y^{-2}\right)\right) .
\end{aligned}
$$

The asymptotics (2.32) can be differentiated any number of times with respect to $y$. Now, we consider $A_{2,0}$ and write it as

$$
A_{2,0}(y)=2 d_{1}^{2} \nu \ln y f_{1}\left(y, \mu_{2}\right)-2(\nu+1) d_{2}^{2} \ln y f_{2}\left(y, \mu_{2}\right)+\widehat{A}_{2,0}(y) .
$$

Then $\widehat{A}_{2,0}(y)$ solves

$$
\left(\mathcal{L}+\mu_{2}\right) \widehat{A}_{2,0}=G
$$


with $G=d_{2}^{2} G_{1}+G_{2}$, where

$$
\begin{aligned}
& G_{1}=-d_{2}^{2}(1+2 \nu)\left(2 y^{-1} \partial_{y}+y^{-2}-i\right) f_{2}\left(y, \mu_{2}\right), \\
& G_{2}=\left|A_{0,0}\right|^{4} A_{0,0}+d_{1}^{2}(1+2 \nu)\left(2 y^{-1} \partial_{y}+y^{-2}\right) f_{1}\left(y, \mu_{2}\right) .
\end{aligned}
$$

The asymptotics (2.30) and (2.32) show that $G_{j}, j=1,2$, has the following behavior as $y \rightarrow+\infty$ :

$$
\begin{aligned}
& G_{1}(y)=e^{i y^{2} / 4} y^{-2 i \alpha_{0}} G_{1,1}(y), \quad G_{2}(y)=\sum_{m=-2}^{3} e^{i m y^{2} / 4} y^{-2 i \alpha_{0} \nu(2 m-1)} G_{2, m}(y), \\
& \partial_{y}^{l} G_{1,1}(y)=O\left(y^{-2+5 \nu-l}\right), \\
& \partial_{y}^{l} G_{2, m}(y)=O\left(y^{-5-5 \nu-|m|(1-2 \nu)-l}\right), \quad-2 \leq m \leq 3,
\end{aligned}
$$

for any $l \geq 0$, provided $\nu$ is sufficiently small.

Integrating (2.34), we get

$$
\widehat{A}_{2,0}(y)=\lambda_{1} f_{1}\left(y, \mu_{2}\right)+\lambda_{2} f_{2}\left(y, \mu_{2}\right)+d_{2}^{2} g_{1}(y)+g_{2}(y) .
$$

Here each $\lambda_{i}, i=1,2$, is a constant, and $g_{i}, i=1,2$, is the solution of $\left(\mathcal{L}+\mu_{2}\right) g_{i}=G_{i}$ with the following behavior as $y \rightarrow+\infty$ :

$$
\begin{aligned}
g_{1}(y) & =e^{i y^{2} / 4} y^{-2 i \alpha_{0}} g_{1,1}(y), \\
g_{2}(y) & =\sum_{m=-2}^{3} e^{i m y^{2} / 4} y^{-2 i \alpha_{0} \nu(2 m-1)} g_{2, m}(y), \\
\partial_{y}^{l} g_{1,1}(y) & =O\left(y^{-2+5 \nu-l}\right), \\
\partial_{y}^{l} g_{2, m}(y) & =O\left(y^{-5-5 \nu-m(1-2 \nu)-l}\right), \quad m=0,1, \\
\partial_{y}^{l} g_{2, m}(y) & =O\left(y^{-7-5 \nu-|m|(1-2 \nu)-l}\right), \quad m=-2,-1,2,3,
\end{aligned}
$$

for any $l \geq 0$.

Denote

$$
\begin{aligned}
& u_{s s}^{a p}(\rho, t)=t^{-(1+2 \nu) / 4} w_{s s}^{a p}\left(t^{-(1+2 \nu) / 2} \rho, t\right), \\
& \chi_{s s}^{a p}(\rho, t)=u_{s s}^{a p}(\rho, t)-W(\rho), \\
& \mathcal{R}_{s s}(\rho, t)=t^{-5(1+2 \nu) / 4} S\left(t^{-(1+2 \nu) / 2} \rho, t\right) .
\end{aligned}
$$

The next lemma is a direct consequence of (2.26), (2.30), (2.32), (2.33), (2.35), and (2.36).

Lemma 2.7. For any $\alpha_{0}, \nu \in \mathbb{R}$ sufficiently small, there exists $T\left(\alpha_{0}, \nu\right)>0$ such that for $t \geq T\left(\alpha_{0}, \nu\right)$ the following holds true.

(i) $\chi_{s s}^{a p}(t)$ verifies

$$
\begin{aligned}
& \left\|\chi_{s s}^{a p}(t)\right\|_{L^{\infty}\left(\frac{1}{10} t^{\frac{1}{2}+\nu-\epsilon_{1}} \leq \rho \leq 10 t^{\frac{1}{2}+\nu+\epsilon_{2}}\right)} \leq C t^{-\frac{1}{2}-\nu}, \\
& \left\|\rho^{-k} \partial_{\rho}^{l} \chi_{s s}^{a p}(t)\right\|_{L^{\infty}\left(\frac{1}{10} t^{\frac{1}{2}+\nu-\epsilon_{1}} \leq \rho \leq 10 t^{\frac{1}{2}+\nu+\epsilon_{2}}\right)} \leq C t^{-1-2 \nu}, \quad k+l=1, \\
& \left\|\rho^{-k} \partial_{\rho}^{l} \chi_{s s}^{a p}(t)\right\|_{L^{\infty}\left(\frac{1}{10} t^{\frac{1}{2}+\nu-\epsilon_{1}} \leq \rho \leq 10 t^{\frac{1}{2}+\nu+\epsilon_{2}}\right)} \leq C\left(\left|\alpha_{0}\right|+|\nu|\right) t^{-1-2 \nu}, \quad k+l=2, \\
& \left\|\rho^{-k} \partial_{\rho}^{l} \chi_{s s}^{a p}(t)\right\|_{L^{2}\left(\rho^{2} d \rho, \frac{1}{10} t^{\frac{1}{2}+\nu-\epsilon_{1}} \leq \rho \leq 10 t^{\left.\frac{1}{2}+\nu+\epsilon_{2}\right)} \leq C t^{-(1+2 \nu)\left(1-2 \varepsilon_{2}\right) / 4},\right.}^{1 \leq k+l \leq 2 .}
\end{aligned}
$$

(ii) The error $\mathcal{R}_{s s}(t)$ admits the estimate

$$
\begin{aligned}
& \left\|\rho^{-k} \partial_{\rho}^{l} \mathcal{R}_{s s}(t)\right\|_{L^{2}\left(\rho^{2} d \rho, \frac{1}{10} t^{\frac{1}{2}+\nu-\epsilon_{1}} \leq \rho \leq 10 t^{\left.\frac{1}{2}+\nu+\epsilon_{2}\right)}\right.} \leq C t^{-\left(2+\frac{1}{4}\right)(1+2 \nu)+5 \varepsilon_{1} / 2}, \\
& 0 \leq k+l \leq 2 \text {. }
\end{aligned}
$$


(iii) The difference $u_{i n}^{a p}(\rho, t)-u_{s s}^{a p}(\rho, t)$ verifies

$$
\left|\partial_{\rho}^{l}\left(u_{i n}^{a p}(t)-u_{s s}^{a p}(t)\right)\right| \leq C \rho^{-2-l} t^{-(1+2 \nu)}\left(\ln t+t^{3(1+2 \nu) / 2-(2 N+3) \varepsilon_{1}}\right),
$$

for any $l \geq 0$ and $\frac{1}{10} t^{\frac{1}{2}+\nu-\epsilon_{1}} \leq \rho \leq 10 t^{\frac{1}{2}+\nu-\epsilon_{1}}$.

2.3. The remote region. Next we consider the remote region $|x| \geq \frac{1}{10} t^{1 / 2+\varepsilon_{2}}$. In this region, as an approximate solution of (1.1) we take the following radial profile:

$$
\psi_{\text {out }}^{a p}(x, t)=v_{1}(x, t)+v_{2}(x, t)+v_{3}(x, t),
$$

where

$$
\begin{aligned}
& v_{1}(x, t)=e^{i \alpha_{0} \ln t}\left[d_{1}^{0} t^{-(1+\nu) / 2} f_{1}\left(y, \mu_{0}\right)+d_{1}^{1} t^{-(2+3 \nu) / 2} f_{1}\left(y, \mu_{1}\right)\right], \quad y=t^{-1 / 2}|x|, \\
& v_{2}(x, t)=\Theta_{\delta}\left(\frac{x}{t}\right) e^{i \alpha_{0} \ln t}\left[d_{2}^{0} t^{-(1+\nu) / 2} f_{2}\left(y, \mu_{0}\right)+d_{2}^{1} t^{-(2+3 \nu) / 2} f_{2}\left(y, \mu_{1}\right)\right. \\
& \left.+t^{-(3+5 \nu) / 2}\left(d_{2}^{2} g_{1}(y)-\left(d_{2}^{2}(2 \nu+1) \ln \left(\frac{|x|}{t}\right)-\lambda_{2}\right) f_{2}\left(y, \mu_{2}\right)\right)\right],
\end{aligned}
$$

$\Theta_{\delta}(\xi)=\Theta\left(\frac{\xi}{\delta}\right), \Theta \in C_{0}^{\infty}\left(\mathbb{R}^{3}\right)$ is radial, $\Theta(\xi)= \begin{cases}1 & \text { if }|\xi| \leq 1, \\ 0 & \text { if }|\xi| \geq 2 .\end{cases}$

Finally, $v_{3}(x, t)$ is given by

$$
v_{3}(x, t)=\frac{e^{i \frac{|x|^{2}}{4 t}}}{t^{5 / 2}} \widehat{v}_{3}\left(\frac{x}{t}\right), \quad \widehat{v}_{3}=-i z \Delta \Theta_{\delta}-2 i \nabla z \cdot \nabla \Theta_{\delta},
$$

where

$$
z(\xi)=d_{2}^{0}|\xi|^{-2 i \alpha_{0}-2+\nu}+d_{2}^{1}|\xi|^{-2 i \alpha_{0}-1+3 \nu}-\left(d_{2}^{2}(2 \nu+1) \ln |\xi|-\lambda_{2}\right)|\xi|^{-2 i \alpha_{0}+5 \nu} .
$$

The asymptotics (2.30) show that, for $t \geq T$ with some $T=T(\delta)>0$ and for any $l \geq 0$, we have

$$
\begin{aligned}
& \left|\nabla^{l} v_{1}(x, t)\right| \leq C_{l}|x|^{-l-1-\nu}, \quad \frac{1}{10} t^{1 / 2+\varepsilon_{2}} \leq|x| \\
& \left|\nabla^{l} v_{2}(x, t)\right| \leq \frac{C_{l}}{t^{3 / 2}}\left|\frac{x}{t}\right|^{l-2+\nu}, \quad \frac{1}{10} t^{1 / 2+\varepsilon_{2}} \leq|x| \leq 2 \delta t .
\end{aligned}
$$

Furthermore, $v_{2}$ can be written as

$$
\begin{aligned}
v_{2}(x, t) & =v_{2,0}(x, t)+v_{2,1}(x, t), \\
v_{2,0}(x, t) & =\frac{e^{i \frac{|x|^{2}}{4 t}}}{t^{3 / 2}} \Theta_{\delta}\left(\frac{x}{t}\right) z\left(\frac{x}{t}\right), \quad v_{2,1}(x, t)=\frac{e^{i \frac{|x|^{2}}{4 t}}}{t^{3 / 2}} \Theta_{\delta}\left(\frac{x}{t}\right) \widehat{v}_{2,1}(x, t),
\end{aligned}
$$

with $\widehat{v}_{2,1}$ verifying

$$
\left|\nabla^{l} \widehat{v}_{2,1}(x, t)\right| \leq C_{l} t^{3-\nu}|x|^{-l-4+\nu}, \quad \frac{1}{10} t^{1 / 2+\varepsilon_{2}} \leq|x| \leq 2 \delta t,
$$

for any $l \geq 0$.

Next we address $v_{3}$. We have

$$
\begin{aligned}
\left\|\nabla^{l} v_{3}(t)\right\|_{L^{\infty}\left(|x| \geq \frac{1}{10} t^{\left.1 / 2+\varepsilon_{2}\right)}\right.} & \leq C_{l} t^{-5 / 2} \delta^{-4+l+\nu}, \\
\left\|\nabla^{l} v_{3}(t)\right\|_{L^{2}\left(|x| \geq \frac{1}{10} t^{\left.1 / 2+\varepsilon_{2}\right)}\right.} & \leq C_{l} t^{-1} \delta^{-5 / 2+l+\nu},
\end{aligned}
$$

for any $l \geq 0$ and $t \geq T(\delta)$. 
As a direct consequence of estimates (2.43), (2.45), (2.46), we obtain

$$
\begin{aligned}
& \left\|\psi_{\text {out }}^{a p}(t)\right\|_{L^{\infty}\left(|x| \geq \frac{1}{10} t^{\left.\frac{1}{2}+\varepsilon_{2}\right)}\right.} \leq C t^{-\left(\frac{1}{2}+\varepsilon_{2}\right)(1+\nu)}, \\
& \left\||x|^{-1} \psi_{\text {out }}^{a p}(t)\right\|_{L^{\infty}\left(|x| \geq \frac{1}{10} t^{\frac{1}{2}+\varepsilon_{2}}\right)} \leq C t^{-5 / 4}, \\
& \left\|\nabla^{l} \psi_{\text {out }}^{a p}(t)\right\|_{L^{\infty}\left(|x| \geq \frac{1}{10} t^{\frac{1}{2}+\varepsilon_{2}}\right)} \leq C t^{-5 / 4}, \quad l=1,2, \\
& \left\|\nabla^{l} \psi_{\text {out }}^{a p}(t)\right\|_{L^{2}\left(|x| \geq \frac{1}{10} t^{\left.\frac{1}{2}+\varepsilon_{2}\right)}\right.} \leq C \delta^{\nu+l-1 / 2}, \quad l=1,2, \\
& \left\|\nabla^{l}\left(\psi_{\text {out }}^{a p}(t)-v_{2,0}(t)\right)\right\|_{L^{2}\left(|x| \geq \frac{1}{10} t^{\frac{1}{2}+\varepsilon_{2}}\right)} \leq C t^{-\frac{1}{2}\left(\frac{1}{2}+\varepsilon_{2}\right)(1+2 \nu)}, \quad l=1,2, \\
& \left\||x|^{-1}\left(\psi_{\text {out }}^{a p}(t)-v_{2,0}(t)\right)\right\|_{L^{2}\left(|x| \geq \frac{1}{10} t^{\frac{1}{2}+\varepsilon_{2}}\right)} \leq C t^{-\frac{1}{2}\left(\frac{1}{2}+\varepsilon_{2}\right)(1+2 \nu)},
\end{aligned}
$$

provided $\frac{3}{8} \leq \varepsilon_{2}<\frac{1}{2}, \nu$ is sufficiently small, and $t \geq T(\delta)$.

Denote

$$
\psi_{s s}^{a p}(x, t)=e^{i \alpha_{0} \ln t} t^{-1 / 4} w_{s s}^{a p}\left(t^{-1 / 2}|x|, t\right),
$$

and consider the difference $\psi_{s s}^{a p}(x, t)-\psi_{\text {out }}^{a p}(x, t)$. If $\frac{1}{10} t^{1 / 2+\varepsilon_{2}} \leq|x| \leq 10 t^{1 / 2+\varepsilon_{2}}$, then

$$
\psi_{s s}^{a p}(x, t)-\psi_{\text {out }}^{a p}(x, t)=e^{i \alpha_{0} \ln t} t^{-(3+5 \nu) / 2}\left(\left(d_{1}^{2}(1+2 \nu) \ln |x|+\lambda_{1}\right) f_{1}\left(y, \mu_{2}\right)+g_{2}(y)\right) \text {, }
$$

which together with (2.30) and (2.36) implies that

$$
\left|\nabla^{l}\left(\psi_{\text {out }}^{a p}-\psi_{s s}^{a p}\right)\right| \leq C_{l}\left(|\ln t| t^{-\left(\frac{1}{2}+\varepsilon_{2}\right)(3+5 \nu+l)}+t^{-\left(\frac{1}{2}+\varepsilon_{2}\right)(3+5 \nu+1)}\right),
$$

for any $l \geq 0$ and $\frac{1}{10} t^{1 / 2+\varepsilon_{2}} \leq|x| \leq 10 t^{1 / 2+\varepsilon_{2}}$, provided $\nu$ is sufficiently small.

Now we analyze the error $R_{\text {out }}(t)=-i \partial_{t} \psi_{\text {out }}^{a p}(t)-\triangle \psi_{\text {out }}^{a p}(t)-\left|\psi_{\text {out }}^{a p}(t)\right|^{4} \psi_{\text {out }}^{a p}(t)$. It has the form

$$
\begin{aligned}
R_{\text {out }}(x, t)= & -\frac{e^{i \frac{|x|^{2}}{4 t}}}{t^{9 / 2}}\left[t \widehat{v}_{2,1}(x, t) \Delta \Theta_{\delta}\left(\frac{x}{t}\right)+2 t^{2} \nabla \widehat{v}_{2,1}(x, t) \cdot \nabla \Theta_{\delta}\left(\frac{x}{t}\right)+\Delta \widehat{v}_{3}\left(\frac{x}{t}\right)\right] \\
& -\left|\psi_{\text {out }}^{a p}\right|^{4} \psi_{\text {out }}^{\text {ap }} .
\end{aligned}
$$

Combined with (2.43), (2.45), (2.46), formula (2.50) shows that if $\frac{3}{8} \leq \varepsilon_{2}<\frac{1}{2}$ and $\nu$ is sufficiently small, then

$$
\left\|\nabla^{l} R_{\text {out }}(t)\right\|_{L^{2}\left(|x| \geq \frac{1}{10} t^{\left.1 / 2+\varepsilon_{2}\right)}\right.} \leq C t^{-\frac{9}{4}(1+2 \nu)}, \quad t \geq T(\delta), \quad l=0,1,2 .
$$

2.4. Proof of Proposition 2.1. Now we are in a position to conclude the proof of Proposition 2.1. Fix $\varepsilon_{2}$ such that $\frac{3}{8} \leq \varepsilon_{2}<\frac{1}{2}$ and consider the radial profile $\psi^{a p}(x, t)$ defined by

$$
\begin{aligned}
\psi^{a p}(x, t)=\Theta\left(t^{-1 / 2+\varepsilon_{1}} x\right) \psi_{i n}^{a p}(x, t) & +\left(1-\Theta\left(t^{-1 / 2+\varepsilon_{1}} x\right)\right) \Theta\left(t^{-1 / 2-\varepsilon_{2}} x\right) \psi_{s s}^{a p}(x, t) \\
& +\left(1-\Theta\left(t^{-1 / 2-\varepsilon_{2}} x\right)\right) \psi_{\text {out }}^{a p}(x, t), \quad x \in \mathbb{R}^{3},
\end{aligned}
$$

where $\psi_{i n}^{a p}(x, t)=e^{i \alpha_{0} \ln t} t^{\nu / 2} u_{i n}^{a p}\left(t^{\nu}|x|, t\right)$. Write $\psi^{a p}$ as

$$
\psi^{a p}(x, t)=e^{i \alpha_{0} \ln t} t^{\nu / 2}\left(W(y)+\chi^{a p}(y, t)\right), \quad y=t^{\nu} x .
$$

By Lemma 2.4 (estimates (2.17), (2.18) ), Lemma 2.7 (estimates (2.37), (2.38), (2.39)), and (2.47), we have

$$
\begin{aligned}
& \left\|\chi^{a p}(t)\right\|_{L^{\infty}} \leq C t^{-(1+2 \nu) / 2} \\
& \left\||y|^{-1} \chi^{a p}(t)\right\|_{L^{\infty}}+\left\|\nabla \chi^{a p}(t)\right\|_{L^{\infty}} \leq C t^{-1-2 \nu} \\
& \left\||y|^{-2} \chi^{a p}(t)\right\|_{L^{\infty}}+\left\||y|^{-1} \nabla_{y} \chi^{a p}(t)\right\|_{L^{\infty}} \leq C\left(|\nu|+\left|\alpha_{0}\right|\right) t^{-1-2 \nu}, \\
& \left\|\nabla^{2} \chi^{a p}(t)\right\|_{L^{\infty}} \leq C\left(|\nu|+\left|\alpha_{0}\right|\right) t^{-1-2 \nu} .
\end{aligned}
$$


All the estimates stated in this subsection are valid for $\nu$ sufficiently small and $t \geq$ $T\left(\alpha_{0}, \nu, \delta\right)$.

Futhermore, from Lemma 2.4 (estimate (2.19) ), Lemma 2.7 (estimate (2.39) ) and the last two inequalities in (2.47) it follows that

$$
\begin{aligned}
& \left\|\nabla^{l} \chi^{a p}(t)\right\|_{L^{2}\left(|y| \leq 10 t^{\left.1 / 2+\nu+\varepsilon_{2}\right)}\right.} \leq C t^{-(1+2 \nu)\left(1-2 \varepsilon_{2}\right) / 4}, \quad l=1,2, \\
& \left\|\nabla^{l}\left(\chi^{a p}(t)-\chi_{0}^{a p}(t)\right)\right\|_{L^{2}\left(|y| \geq t^{\left.1 / 2+\nu+\varepsilon_{2}\right)}\right.} \leq C t^{-(1+2 \nu) / 4}, \quad l=1,2,
\end{aligned}
$$

where $\chi_{0}^{a p}(y, t)=e^{-i \alpha_{0} \ln t} t^{-\nu / 2} v_{2,0}\left(t^{-\nu} y, t\right)$.

In particular, inequalities (2.56) imply

$$
\left\|\nabla^{l} \chi^{a p}(t)\right\|_{L^{2}\left(\mathbb{R}^{3}\right)} \leq C t^{-\nu(l-1)} \delta^{\nu+l-1 / 2}, \quad l=1,2 .
$$

Moreover, introducing $\zeta^{*}(x)=\pi^{-3 / 2} e^{3 i \pi / 4} \int_{\mathbb{R}^{3}} d \xi e^{i x \cdot \xi} \Theta_{\delta}(2 \xi) z(2 \xi)$ and observing that $\zeta^{*} \in \dot{H}^{s}\left(\mathbb{R}^{3}\right)$ for any $s>1 / 2-\nu$, and $\left\|\nabla^{l}\left(v_{2,0}-e^{i \Delta t} \zeta^{*}\right)\right\|_{L^{2}\left(|x| \geq t^{\gamma}\right)} \rightarrow 0$ as $t \rightarrow+\infty$ for any $\gamma>\frac{1-2 \nu}{3-2 \nu}$ and any $l \geq 1$, we get that

$$
e^{i \alpha(t)} \lambda^{1 / 2}(t) \chi^{a p}(\lambda(t) \cdot, t)-e^{i t \Delta} \zeta^{*} \rightarrow 0 \text { in } \dot{H}^{1} \cap \dot{H}^{2} \text { as } t \rightarrow+\infty .
$$

This concludes the proof of the first part of Proposition 2.1.

Next, we consider the error $R=-i \psi_{t}^{a p}-\Delta \psi^{a p}-\left|\psi^{a p}\right|^{4} \psi^{a p}$. It has the form

$$
R=E_{1}+E_{2}+E_{3}+E_{4},
$$

where

$$
\begin{aligned}
E_{1}=i( & \left(\frac{1}{2}-\varepsilon_{1}\right) t^{-1}\left(\psi_{i n}^{a p}(x, t)-\psi_{s s}^{a p}(x, t)\right) \widetilde{\Theta}\left(t^{-1 / 2+\varepsilon_{1}} x\right) \\
& -2 t^{-1 / 2+\varepsilon_{1}}\left(\nabla \psi_{i n}^{a p}(x, t)-\nabla \psi_{s s}^{a p}(x, t)\right) \cdot \nabla \Theta\left(t^{-1 / 2+\varepsilon_{1}} x\right) \\
& -t^{-1+2 \varepsilon_{1}}\left(\psi_{i n}^{a p}(x, t)-\psi_{s s}^{a p}(x, t)\right) \Delta \Theta\left(t^{-1 / 2+\varepsilon_{1}} x\right), \\
E_{2}=i( & \left.\frac{1}{2}+\varepsilon_{2}\right) t^{-1}\left(\psi_{s s}^{a p}(x, t)-\psi_{\text {out }}^{a p}(x, t)\right) \widetilde{\Theta}\left(t^{-1 / 2-\varepsilon_{2}} x\right) \\
& -2 t^{-1 / 2-\varepsilon_{2}}\left(\nabla \psi_{s s}^{a p}(x, t)-\nabla \psi_{\text {out }}^{a p}(x, t)\right) \cdot \nabla \Theta\left(t^{-1 / 2-\varepsilon_{2}} x\right) \\
& -t^{-1-2 \varepsilon_{2}}\left(\psi_{s s}^{a p}(x, t)-\psi_{\text {out }}^{a p}(x, t)\right) \Delta \Theta\left(t^{-1 / 2-\varepsilon_{2}} x\right), \\
\widetilde{\Theta}(\xi)=\xi & \cdot \nabla \Theta(\xi),
\end{aligned}
$$

and $E_{3}, E_{4}$ are given by

$$
\begin{aligned}
E_{3}=\Theta & \left(t^{-1 / 2+\varepsilon_{1}} x\right) R_{\text {in }}(x, t)+\left(1-\Theta\left(t^{-1 / 2+\varepsilon_{1}} x\right)\right) \Theta\left(t^{-1 / 2-\varepsilon_{2}} x\right) R_{s s}(x, t) \\
& +\left(1-\Theta\left(t^{-1 / 2-\varepsilon_{2}} x\right)\right) R_{\text {out }}(x, t), \\
E_{4}=\Theta & \left(t^{-1 / 2+\varepsilon_{1}} x\right)\left(\left|\psi_{\text {in }}^{a p}\right|^{4} \psi_{\text {in }}^{a p}-\left|\psi^{a p}\right|^{4} \psi^{a p}\right) \\
& +\left(1-\Theta\left(t^{-1 / 2+\varepsilon_{1}} x\right)\right) \Theta\left(t^{-1 / 2-\varepsilon_{2}} x\right)\left(\left|\psi_{s s}^{a p}\right|^{4} \psi_{s s}^{a p}-\left|\psi^{a p}\right|^{4} \psi^{a p}\right) \\
& +\left(1-\Theta\left(t^{-1 / 2-\varepsilon_{2}} x\right)\right)\left(\left|\psi_{\text {out }}^{a p}\right|^{4} \psi_{\text {out }}^{a p}-\left|\psi^{a p}\right|^{4} \psi^{a p}\right) .
\end{aligned}
$$

Here

$$
R_{i n}(x, t)=e^{i \alpha_{0} \ln t} t^{5 \nu / 2} \mathcal{R}_{i n}\left(t^{\nu}|x|, t\right), \quad R_{s s}(x, t)=e^{i \alpha_{0} \ln t} t^{5 \nu / 2} \mathcal{R}_{s s}\left(t^{\nu}|x|, t\right) .
$$

First, we address $E_{1}$. By Lemma 2.7 (iii), we have

$$
\left\|E_{1}\right\|_{H^{2}} \leq C t^{-9(1+2 \nu) / 4+\nu+5 \varepsilon_{1} / 2} \ln t \leq C t^{-\left(2+\frac{3}{20}\right)(1+2 \nu)} .
$$

Similarly, by (2.49), for $E_{2}$ we get

$$
\left\|E_{2}\right\|_{H^{2}} \leq C t^{-1-\left(\frac{1}{2}+\varepsilon_{2}\right)\left(\frac{3}{2}+5 \nu\right)} \ln t \leq C t^{-\left(2+\frac{1}{4}\right)(1+2 \nu)} .
$$


Next, consider $E_{3}$. From Lemma 2.4 (ii), Lemma 2.7 (ii), and (2.51) it is clear that

$$
\left\|E_{3}\right\|_{H^{2}} \leq C t^{-\frac{9}{4}(1+2 \nu)+5 \varepsilon_{1} / 2} \leq C t^{-\left(2+\frac{3}{20}\right)(1+2 \nu)} .
$$

Finally, applying Lemma 2.4 (estimates (2.17), (2.18)), Lemma 2.7 (estimates (2.37), (2.38), (2.39), (2.42) $)$, and (2.47), (2.49), it is not difficult to check that

$$
\left\|E_{4}\right\|_{H^{2}} \leq C t^{-3(1+2 \nu)}
$$

Combining (2.57), (2.58), (2.59), (2.60), we get (2.6), which concludes the proof of Proposition 2.1 .

\section{§3. Construction of an exact solution}

Now we are in a position to prove Theorem 1.1 Consider (1.1) and write $\psi(x, t)=$ $e^{i \alpha_{0} \ln t} t^{\nu / 2} U(y, \tau)$, where $y=t^{\nu} x$ and $\tau=\frac{t^{1+2 \nu}}{1+2 \nu}$. Further decomposing $U$ as

$$
U(y, \tau)=U^{a p}(y, \tau)+f(y, \tau), \quad U^{a p}(y, \tau)=e^{-i \alpha_{0} \ln t} t^{-\nu / 2} \psi^{a p}(x, t),
$$

where $\psi^{a p}$ is the approximate solution of (1.1) given by Proposition 2.1, we get the following equation for the remainder $f$ :

$$
i \overrightarrow{f_{\tau}}=\mathcal{H}(\tau) \vec{f}+\mathcal{F}(f)+r, \quad \vec{f}=\left(\begin{array}{l}
f \\
\bar{f}
\end{array}\right)
$$

where

$$
\begin{aligned}
& \mathcal{H}(\tau)=H+\tau^{-1} l \\
& H=-\triangle \sigma_{3}-3 W^{4} \sigma_{3}-2 W^{4} \sigma_{3} \sigma_{1}, \quad l=\frac{\alpha_{0}}{2 \nu+1} \sigma_{3}-i \frac{\nu}{2 \nu+1}\left(\frac{1}{2}+y \cdot \nabla\right), \\
& \mathcal{F}(f)=\left(\begin{array}{c}
F(f) \\
-\overline{F(f)}
\end{array}\right), \quad F(f)=F_{1}(f)+F_{2}(f), \\
& F_{1}(f)=\mathcal{V}_{1}(\tau) f+\mathcal{V}_{2}(\tau) \bar{f} \\
& \mathcal{V}_{1}(\tau)=3\left(W^{4}-\left|U^{a p}(\tau)\right|^{4}\right), \quad \mathcal{V}_{2}(\tau)=2\left(W^{4}-\left(U^{a p}(\tau)\right)^{2}\left|U^{a p}(\tau)\right|^{2}\right), \\
& F_{2}(f)=-\left|U^{a p}+f\right|^{4}\left(U^{a p}+f\right)+\left|U^{a p}\right|^{4} U^{a p}+3\left|U^{a p}\right|^{4} f+2\left(U^{a p}\right)^{2}\left|U^{a p}\right|^{2} \bar{f}, \\
& r=\left(\begin{array}{c}
\mathrm{r} \\
-\overline{\mathrm{r}}
\end{array}\right), \quad \mathrm{r}(y, \tau)=t^{-5 \nu / 2} e^{-i \alpha_{0} \ln t} R(x, t),
\end{aligned}
$$

$R$ being the error given by Proposition 2.1. Note that by Proposition 2.1 we have

$$
\begin{aligned}
& \left\|\mathcal{V}_{i}(\tau)\right\|_{W^{2, \infty}\left(\mathbb{R}^{3}\right)} \leq C\left(\left|\alpha_{0}\right|+|\nu|\right) \tau^{-1}, \quad i=1,2, \\
& \left\|U^{a p}(\tau)\right\|_{W^{2, \infty}\left(\mathbb{R}^{3}\right)} \leq C, \\
& \|r(\tau)\|_{H^{2}\left(\mathbb{R}^{3}\right)} \leq C \tau^{-2-\frac{1}{8}},
\end{aligned}
$$

for any $\tau \geq \tau_{0}$ with some $\tau_{0}>0$.

Our intention is to solve (3.1) with zero condition at $\tau=+\infty$ by a fixed point argument. To carry out this analysis, we shall need some energy type estimates for the linearized equation $i \vec{f}_{\tau}=\mathcal{H}(\tau) \vec{f}$. The required estimates are collected in the next subsection, their proofs can be found in $\S 4$. 
3.1. Linear estimates. We start by recalling some basic spectral properties of the operator $H$ (see, e.g., [5] for a more detailed discussion and proofs). Since we are considering only radial solutions, we shall view $H$ as an operator on $L_{\text {rad }}^{2}\left(\mathbb{R}^{3} ; \mathbb{C}^{2}\right)$ with domain $D(H)=H_{\text {rad }}^{2}\left(\mathbb{R}^{3} ; \mathbb{C}^{3}\right) . H$ satisfies the relations

$$
\sigma_{3} H \sigma_{3}=H^{*}, \quad \sigma_{1} H \sigma_{1}=-H .
$$

The essential spectrum of $H$ fills up the real axis. The discrete spectrum of $H$ consists of two simple purely imaginary eigenvalues $i \lambda_{0},-i \lambda_{0}, \lambda_{0}>0$. The corresponding eigenfunctions $\zeta_{+}, \zeta_{-}$are in $\mathcal{S}\left(\mathbb{R}^{3}\right)$ and can be chosen in such a way that $\zeta_{-}=\sigma_{1} \zeta_{+}=\bar{\zeta}_{+}$. Observe also that $H W\left(\begin{array}{c}1 \\ -1\end{array}\right)=H W_{1}\left(\begin{array}{l}1 \\ 1\end{array}\right)=0$, which means that $H$ has a resonance at zero.

Consider the projection of the linearized equation $i \vec{f}_{\tau}=\mathcal{H}(\tau) \vec{f}$ to the essential spectrum of $H$ :

$$
i \overrightarrow{f_{\tau}}=P \mathcal{H}(\tau) P \vec{f}
$$

Here $P$ is the spectral projection of $H$ to the essential spectrum given by

$$
P=I-P_{+}-P_{-}, \quad P_{ \pm}=\frac{\left\langle\cdot, \sigma_{3} \zeta_{\mp}\right\rangle}{\left\langle\zeta_{ \pm}, \sigma_{3} \zeta_{\mp}\right\rangle} \zeta_{ \pm},
$$

$\langle\cdot, \cdot\rangle$ is the scalar product in $L^{2}\left(\mathbb{R}^{3}, \mathbb{C}^{2}\right)$.

Let $U(\tau, s)$ be the propagator associated with equation (3.5). In $\S 4$ we prove the following results.

Proposition 3.1. There exists a constant $C>0$ such that

$$
\|U(\tau, s) f\|_{H^{2}} \leq C\left(\frac{s}{\tau}\right)^{C\left(\left|\alpha_{1}\right|+\left|\nu_{1}\right|\right)}\|f\|_{H^{2}}
$$

for any $s \geq \tau>0$ and any $f \in H_{\mathrm{rad}}^{2}$. Here $\alpha_{1}=\frac{\alpha_{0}}{1+2 \nu}, \nu_{1}=\frac{\nu}{1+2 \nu}$.

3.2. Contraction argument. Now we transform (3.1) into a fixed point problem. Rewrite (3.1) in the following integral form:

$$
f(\tau)=J(f)(\tau),
$$

where

$$
\begin{aligned}
& J(f)(\tau)=J_{0}(f)(\tau)+J_{+}(f)(\tau)+J_{-}(f)(\tau), \\
& J_{0}(f)(\tau)=i \int_{\tau}^{+\infty} d s U(\tau, s) P\left(\mathcal{F}_{1}(f(s))+r(s)\right), \\
& J_{+}(f)(\tau)=i \int_{\tau}^{+\infty} d s e^{\lambda_{0}(\tau-s)} P_{+}\left(\mathcal{F}_{2}(f(s))+r(s)\right), \\
& J_{-}(f)(\tau)=-i \int_{\tau_{1}}^{\tau} d s e^{-\lambda_{0}(\tau-s)} P_{-}\left(\mathcal{F}_{2}(f(s))+r(s)\right), \\
& \mathcal{F}_{1}(f)=\mathcal{F}(f)+s^{-1} l\left(P_{+}+P_{-}\right) \vec{f}, \\
& \mathcal{F}_{2}(f)=\mathcal{F}(f)+s^{-1} l \vec{f},
\end{aligned}
$$

with $\tau_{1} \geq \max \left\{\tau_{0}, 1\right\}$ to be fixed later (slightly abusing notation, in (3.6) we identify $\mathbb{C}^{2}$-vectors of the form $\left(\frac{f}{\bar{f}}\right)$ with their first component $f$ ).

Our intention is to view $J$ as a mapping in the space $C\left(\left[\tau_{1},+\infty\right), H_{\text {rad }}^{2}\right)$ equipped with the norm $\||f|\|=\sup _{\tau \geq \tau_{1}}\|f(\tau)\|_{H^{2}} \tau^{1+1 / 16}$, and to show that $J$ is a contraction of the unite ball $\||f|\| \leq 1$ into itself provided $\left|\alpha_{0}\right|+|\nu|$ is sufficiently small and $\tau_{1}$ is chosen 
sufficiently large. Indeed, by (3.3) and (3.2) we have, for any $f, g \in H^{2}$ with $\|f\|_{H^{2}} \leq 1$, $\|g\|_{H^{2}} \leq 1$

$$
\begin{aligned}
\left\|\mathcal{F}_{1}(f)-\mathcal{F}_{1}(g)\right\|_{H^{2}} & \leq C\left(\|f\|_{H^{2}}+\|g\|_{H^{2}}+\left(\left|\alpha_{0}\right|+|\nu|\right) \tau^{-1}\right)\|f-g\|_{H^{2}} \\
\left\|P_{ \pm}\left(\mathcal{F}_{2}(f)-\mathcal{F}_{2}(g)\right)\right\| & \leq C\left(\|f\|_{H^{2}}+\|g\|_{H^{2}}+\left(\left|\alpha_{0}\right|+|\nu|\right) \tau^{-1}\right)\|f-g\|_{H^{2}}
\end{aligned}
$$

which, together with (3.4) and Proposition 3.1. gives

$$
\||J(f)|\| \leq \frac{1}{2}+C \tau_{1}^{-1 / 16}, \quad\||J(f)-J(g)|\| \leq\left(\frac{1}{2}+C \tau_{1}^{-1 / 16}\right)\||f-g|\|
$$

for any $f, g \in\{\||h|\| \leq 1\}$, provided $\left|\alpha_{0}\right|+|\nu|$ is sufficiently small. This means that if $\tau_{1}$ is sufficiently large, then $J$ is a contraction of the unit ball $\||f|\| \leq 1$ into itself, and consequently, has a unique fixed point $f$ satisfying

$$
\|f(\tau)\|_{H^{2}} \leq \tau^{-1-1 / 16}, \text { for all } \tau \geq \tau_{1} .
$$

Together with Proposition 2.1, this gives Theorem 1.1

\section{§4. Linearized EVOLUtion}

In this section we prove Proposition 3.1. The proof will be achieved by combining the results of [5] with a careful spectral analysis of the operator $H$ around the zero energy. The section is organized as follows. In Subsection 1 we consider the operator $H$ as before, restricted to the subspace of radial functions, and construct a basis of Jost solutions of the equation $H \zeta=E \zeta$. In Subsection 2 we study the spectral decomposition of $H$ near $E=0$. In Subsection 3 we prove Proposition 3.1 by combining the results of the previous two subsections with the coercivity properties of $H$ established in [5].

4.1. Solutions of the equation $H \zeta=E \zeta$. In this subsection we construct a basis of Jost solutions of the equation $H \zeta=E \zeta, E \in \mathbb{R}$. Since the subject is completely standard we shall only briefly scetch the proofs (see also [1] and 7] for a closely related construction in the context of energy subcitical NLS). Recall that

$$
\begin{gathered}
H=-\left(\partial_{\rho}^{2}+2 \rho^{-1} \partial_{\rho}\right) \sigma_{3}+V(\rho), \quad V=\left(\begin{array}{cc}
V_{1} & V_{2} \\
-V_{2} & -V_{1}
\end{array}\right), \\
V_{1}(\rho)=-3 W^{4}(\rho), \quad V_{2}(\rho)=-2 W^{4}(\rho), \quad W(\rho)=\left(1+\rho^{2} / 3\right)^{-1 / 2} .
\end{gathered}
$$

We emphasize that $V(\rho)$ is a smooth function of $\rho$ that decays as $\rho^{-4}$ as $\rho \rightarrow \infty$. Since $\sigma_{1} H=-H \sigma_{1}$, it suffices to consider the case where $E \geq 0$, so we write $E=k^{2}, k \geq 0$. It will be convenient for us to remove the first derivative in $H$. Setting $f=\rho \zeta$, we get

$$
\widetilde{H} f=E f, \quad \widetilde{H}=-\partial_{\rho}^{2} \sigma_{3}+V(\rho) .
$$

We shall consider the operator $\widetilde{H}$ on $\mathbb{R}$; to recover the original radial $\mathbb{R}^{3}$ problem, it suffices to restrict $\widetilde{H}$ to the subspace of odd functions.

We start with constructing the most rapidly decaying solution of (4.1).

Lemma 4.1. For all $k \geq 0$, there exists a real solution $f_{3}(\rho, k)$ of the equation

$$
\widetilde{H} f=k^{2} f
$$

such that $f_{3}(\rho, k)=e^{-k \rho} \chi_{3}(\rho, k)$, where $\chi_{3}$ is $C^{\infty}$-function of $(\rho, k) \in \mathbb{R} \times \mathbb{R}_{+}^{*}$ verifying $\chi_{3}(\rho, k)=\left(\begin{array}{l}0 \\ 1\end{array}\right)+a(\rho, k)$,

$$
\begin{gathered}
\left|\partial_{\rho}^{l} \partial_{k}^{m} a(\rho, k)\right| \leq C_{l}\langle\rho\rangle^{-2-l+m}(1+k\langle\rho\rangle)^{-1-m}, \quad m=0,1, \\
\left|\partial_{\rho}^{l} \partial_{k}^{2} a(\rho, k)\right| \leq C_{l}\langle\rho\rangle^{-l}(1+k\langle\rho\rangle)^{-3} \ln \left(\frac{1}{k\langle\rho\rangle}+2\right),
\end{gathered}
$$

for all $\rho \geq 0, k>0$, and $l \geq 0$. 
Proof. We write the following integral equation for $\chi_{3}$ :

$$
\begin{aligned}
\chi_{3}(\rho, k) & =\left(\begin{array}{l}
0 \\
1
\end{array}\right)-\int_{\rho}^{+\infty} K(\rho-s, k) \sigma_{3} V(s) \chi_{3}(s, k) d s \\
K(\xi, k) & =\left(\begin{array}{cc}
\frac{\sin k \xi}{k} & 0 \\
0 & \frac{\sinh k \xi}{k}
\end{array}\right) e^{k \xi} .
\end{aligned}
$$

Then, the claim follows from the estimate

$$
\left|\partial_{k}^{l} K(\xi, k)\right| \leq C_{l} \frac{|\xi|^{l+1}}{\langle k \xi\rangle^{l+1}}, \quad \xi \leq 0, \quad k \geq 0, \quad l \geq 0
$$

and the decay properties of $V$ :

$$
\left|\partial_{\rho}^{l} V(\rho)\right| \leq C_{l}\langle\rho\rangle^{-4-l}, \quad \rho \in \mathbb{R}, \quad l \geq 0,
$$

by the standard Volterra iterations.

Next we construct oscillating solutions of (4.2).

Lemma 4.2. For all $k \geq 0$, there exists a solution $f_{1}(\rho, k)$ of equation (4.2) such that $f_{1}$ is a smooth function of $(\rho, k) \in \mathbb{R} \times \mathbb{R}_{+}^{*}$ of the form $f_{1}(\rho, k)=e^{i k \rho}\left(\left(\begin{array}{l}1 \\ 0\end{array}\right)+b(\rho, k)\right)$, where $b$ verifies

$$
\begin{aligned}
|b(\rho, k)| & \leq C\left(\langle\rho\rangle^{-2}+k e^{-k \rho}\right), \\
\left|\partial_{\rho} b(\rho, k)\right| & \leq C\left(\langle\rho\rangle^{-3}+k^{2} e^{-k \rho}\right), \\
\left|\partial_{k} b(\rho, k)\right| & \leq C\left(\langle\rho\rangle^{-1}+\langle k \rho\rangle e^{-k \rho}\right), \\
\left|\partial_{\rho k}^{2} b(\rho, k)\right| & \leq C\left(\langle\rho\rangle^{-2}+k\langle k \rho\rangle e^{-k \rho}\right),
\end{aligned}
$$

for all $\rho \geq 0,0 \leq k \lesssim 1$. Moreover, we have

$$
\left|\partial_{k}^{2} b(\rho, k)\right| \leq C \ln \left(\frac{1}{k}+1\right)
$$

for all $0 \leq \rho \lesssim 1,0<k \lesssim 1$

Proof. To construct $f_{1}$ we reduce the order of system (4.2) via the substitution $f_{1}=$ $z_{0} f_{3}+z_{1}\left(\begin{array}{l}1 \\ 0\end{array}\right)$. Further setting $z_{2}=z_{0}^{\prime} f_{3,2}, f_{3}=\left(\begin{array}{l}f_{3,1} \\ f_{3,2}\end{array}\right)$, we see that $z=\left(\begin{array}{l}z_{1} \\ z_{2}\end{array}\right)$ solves

$$
\begin{aligned}
-z_{1}^{\prime \prime}-k^{2} z_{1}+V_{11} z_{1}+V_{12} z_{2} & =0 \\
-z_{2}^{\prime}+k z_{2}+V_{21} z_{1}+V_{22} z_{2} & =0 .
\end{aligned}
$$

Here

$$
\begin{aligned}
& V_{11}=V_{1}-V_{2} \frac{f_{3,1}}{f_{3,2}}, \quad V_{12}=\frac{2}{f_{3,2}^{2}}\left(f_{3,1} f_{3,2}^{\prime}-f_{3,1}^{\prime} f_{3,2}\right), \\
& V_{21}=V_{2}, \quad V_{22}=-\frac{1}{f_{3,2}}\left(f_{3,2}^{\prime}+k f_{3,2}\right) .
\end{aligned}
$$

By Lemma 4.1, there exists $R>0$ independent of $k$ such that the functions $V_{i j}(\rho, k)$, $i, j=1,2$, are smooth in both variables for $k>0$ and $\rho \geq R$ and verify, for all $l \geq 0$, 
$\rho \geq R$, and $k>0$

$$
\begin{aligned}
\left|\partial_{\rho}^{l} V_{j 1}(\rho, k)\right| & \leq C_{l}\langle\rho\rangle^{-4-l}, \quad j=1,2, \\
\left|\partial_{\rho}^{l} \partial_{k} V_{11}(\rho, k)\right| & \leq C_{l}\langle\rho\rangle^{-5-l}\langle k \rho\rangle^{-2}, \\
\left|\partial_{\rho}^{l} \partial_{k}^{2} V_{11}(\rho, k)\right| & \leq C_{l}\langle\rho\rangle^{-4-l}\langle k \rho\rangle^{-3} \ln \left(\frac{1}{k \rho}+2\right), \\
\left|\partial_{\rho}^{l} \partial_{k}^{m} V_{j 2}(\rho, k)\right| & \leq C_{l}\langle\rho\rangle^{-3-l+m}\langle k \rho\rangle^{-1-m}, \quad j=1,2, \quad m=0,1, \\
\left|\partial_{\rho}^{l} \partial_{k}^{2} V_{22}(\rho, k)\right| & \leq C_{l}\langle\rho\rangle^{-1-l}\langle k \rho\rangle^{-3} \ln \left(\frac{1}{k \rho}+2\right) .
\end{aligned}
$$

Writing for $z$ the integral equation

$$
z(\rho, k)=e^{i k \rho}\left(\begin{array}{l}
1 \\
0
\end{array}\right)-\int_{\rho}^{\infty}\left(\begin{array}{cc}
\frac{\sin k(\rho-s)}{k} & 0 \\
0 & e^{-k(s-\rho)}
\end{array}\right)\left(\begin{array}{ll}
V_{11} & V_{12} \\
V_{21} & V_{22}
\end{array}\right) z(s, k) d s,
$$

and taking (4.6) into account, we easily prove the existence of a smooth solution satisfying

$$
\begin{gathered}
\left|\partial_{\rho}^{l} \partial_{k}^{m}\left(e^{-i k \rho} z_{1}-1\right)\right|+\langle\rho\rangle\left|\partial_{\rho}^{l} \partial_{k}^{m}\left(e^{-i k \rho} z_{2}\right)\right| \leq C_{l}\langle\rho\rangle^{-2-l+m}\langle k \rho\rangle^{-1-m}, \quad m=0,1, \\
\left|\partial_{\rho}^{n} \partial_{k}^{2}\left(e^{-i k \rho} z_{1}-1\right)\right|+\left|\partial_{\rho}^{n} \partial_{k}^{2}\left(e^{-i k \rho} z_{2}\right)\right| \leq C \ln \left(\frac{1}{k \rho}+2\right), \quad n=0,1,
\end{gathered}
$$

for all $\rho \geq R, k>0, l \geq 0$.

To recover $f_{1}$, we set

$$
z_{0}(\rho, k)=\int_{R}^{\rho} \frac{z_{2}(s, k)}{f_{3,2}(s, k)} d s-\int_{R}^{+\infty} \frac{z_{2}(s, 0)}{f_{3,2}(s, 0)} d s .
$$

Then, for $\rho \geq R$, the claim of Lemma 4.2 follows directly from (4.7) and Lemma 4.1. To cover the case where $x \leq R$, we can invoke the Cauchy problem with initial data at $\rho=R$.

Note that, since $k^{2} \in \mathbb{R}$, the function $f_{2}(\cdot, k)=\overline{f_{1}(\cdot, k)}$ is also a solution of (4.2).

Remark 4.3. Recall that the equation $\widetilde{H} f=0$ has a basis of explicit solutions $\rho \Phi_{ \pm}(\rho)\left(\begin{array}{c}1 \\ \pm 1\end{array}\right)$, $\rho \Theta_{ \pm}(\rho)\left(\begin{array}{c}1 \\ \pm 1\end{array}\right)$, with $\Phi_{ \pm}, \Theta_{ \pm}$given by (2.14). Comparing the behavior of $\rho \Phi_{ \pm}, \rho \Theta_{ \pm}$with the asymptotics of $f_{1}(\rho, 0), f_{3}(\rho, 0)$, we see that

$$
f_{1}(\rho, 0)=\frac{1}{2} \rho\left(\xi_{0}(\rho)+\xi_{1}(\rho)\right), \quad f_{3}(\rho, 0)=\frac{1}{2} \rho\left(\xi_{1}(\rho)-\xi_{0}(\rho)\right),
$$

where $\xi_{0}=\frac{1}{\sqrt{3}} W\left(\begin{array}{c}1 \\ -1\end{array}\right), \xi_{1}=-\frac{2}{\sqrt{3}} W_{1}\left(\begin{array}{l}1 \\ 1\end{array}\right)$.

Next, we construct an exponentially growing solution at $+\infty$.

Lemma 4.4. For any $k>0$, there exists a solution $f_{4}(\rho, k)$ of (4.2) such that $f_{4}=e^{k \rho} \chi_{4}$ with $\chi_{4}$ verifying

$$
\partial_{\rho}^{l}\left(\chi_{4}(\rho, k)-\left(\begin{array}{l}
0 \\
1
\end{array}\right)\right)=O_{k}\left(\rho^{-3-l}\right), \quad \rho \rightarrow+\infty .
$$

Proof. We construct $f_{4}$ with the help of the following integral equation:

$$
\begin{aligned}
\chi_{4}(\rho, k) & =\left(\begin{array}{l}
0 \\
1
\end{array}\right)+\int_{\rho}^{+\infty}\left(\begin{array}{cc}
0 & 0 \\
0 & \frac{1}{2 k}
\end{array}\right) V \chi_{4}(s, k) d s \\
& +\int_{R_{1}}^{\rho}\left(\begin{array}{cc}
\frac{e^{k(s-\rho)} \sin k(\rho-s)}{k} & 0 \\
0 & \frac{e^{2 k(s-\rho)}}{2 k}
\end{array}\right) V \chi_{4}(s, k) d s .
\end{aligned}
$$

For $k>0$ and $R_{1}$ sufficiently large (depending on $k$ ), the operator generating (4.9) is small on the space of bounded continuous functions. Therefore, (4.9) has a solution $\chi_{4}$ verifying 
$\left|\chi_{4}(\rho, k)\right| \leq C, \rho \geq R_{1}$. Iterating this bound, we see that $\chi_{4}(\rho, k)-\left(\begin{array}{l}0 \\ 1\end{array}\right)=O_{k}\left(\rho^{-3}\right)$ as $\rho \rightarrow \infty$. Finally, estimates for the derivatives can be obtained by differentiating (4.9).

Now we briefly describe some properties of the solutions $f_{j}, j=1, \ldots, 4$, that we will need later. Recall that the Wronskian $w(f, g)=\left\langle f^{\prime}, g\right\rangle_{\mathbb{R}^{2}}-\left\langle f, g^{\prime}\right\rangle_{\mathbb{R}^{2}}$ does not depend on $\rho$ if $f$ and $g$ are solutions of (4.1).

The estimates of Lemmas 4.1, 4.2, 4.4 lead to the relations

$$
w\left(f_{1}, f_{2}\right)=2 i k, \quad w\left(f_{1}, f_{3}\right)=w\left(f_{2}, f_{3}\right)=0, \quad w\left(f_{3}, f_{4}\right)=-2 k, \quad k>0,
$$

the three first relations being valid also for $k=0$. Observe that, by Lemmas 4.1 and 4.2 , $\partial_{k} f_{1}(\rho, 0), \partial_{k} f_{3}(\rho, 0)$ are solutions of the equation $\widetilde{H} f=0$ verifying

$$
\begin{aligned}
\left|\partial_{k} f_{1}(\rho, 0)-\left(\begin{array}{c}
i \rho \\
0
\end{array}\right)\right| \leq C, \quad\left|\partial_{k \rho}^{2} f_{1}(\rho, 0)-\left(\begin{array}{l}
i \\
0
\end{array}\right)\right| \leq \frac{C}{\langle\rho\rangle^{2}} \\
\left|\partial_{k} f_{3}(\rho, 0)+\left(\begin{array}{l}
0 \\
\rho
\end{array}\right)\right| \leq \frac{C}{\langle\rho\rangle}, \quad\left|\partial_{k \rho}^{2} \zeta_{3}(\rho, 0)+\left(\begin{array}{l}
0 \\
1
\end{array}\right)\right| \leq \frac{C}{\langle\rho\rangle^{2}}
\end{aligned}
$$

for $\rho \geq 0$. As a consequence,

$$
\begin{array}{ll}
w\left(\left.\partial_{k} f_{1}\right|_{k=0},\left.f_{1}\right|_{k=0}\right)=i, & w\left(\left.\partial_{k} f_{1}\right|_{k=0},\left.f_{3}\right|_{k=0}\right)=0, \\
w\left(\left.\partial_{k} f_{3}\right|_{k=0},\left.f_{1}\right|_{k=0}\right)=0, & w\left(\left.\partial_{k} f_{3}\right|_{k=0},\left.f_{3}\right|_{k=0}\right)=-1 .
\end{array}
$$

In addition to the scalar Wronskian, we shall use matrix Wronskians. If $F, G$ are $(2 \times 2)$ matrix solutions of (4.2), their matrix Wronskian

$$
W(F, G)=F^{t^{\prime}} G-F^{t} G^{\prime}
$$

is independent of $\rho$.

Set $g_{j}(\rho, k)=f_{j}(-\rho, k), j=1, \ldots, 4$. Since the potential $V$ is even, $g_{j}, j=1, \ldots, 4$, are again solutions of (4.2), and, as $\rho \rightarrow-\infty$, they have the same asymptotic behavior as $f_{j}$ as $\rho \rightarrow+\infty$.

Consider the matrix solutions $F, G$ defined by

$$
F=\left(f_{1}, f_{3}\right), \quad G=\left(g_{1}, g_{3}\right) .
$$

Denote $D(k)=W(F, G)$. From Lemmas 4.1, 4.2 it follows that $D$ is smooth for $k>0$ and admits the estimate

$$
\left|\partial_{k}^{2} D(k)\right| \leq C \ln \left(\frac{1}{k}+1\right), \quad 0<k \lesssim 1
$$

Moreover, by (4.8), (4.10), and (4.11) we have

$$
D(0)=0, \quad \partial_{k} D(0)=\left(\begin{array}{cc}
-2 i & 0 \\
0 & 2
\end{array}\right) .
$$

\subsection{Scattering solutions and the distorted Fourier transform in the vicinity of zero energy. Set}

$$
\mathcal{F}(\rho, k)=F(\rho, k) s(k),
$$

where $s(k)=D^{t^{-1}}(k)\left(\begin{array}{c}2 i k \\ 0\end{array}\right)$. By (4.12) and (4.13), $s=\left(\begin{array}{l}s_{1} \\ s_{2}\end{array}\right)$ is a smooth function of $k$ for $0<k<k_{0}$ ( $k_{0}$ sufficiently small), continuous up to $k=0$, and verifying

$$
\begin{aligned}
& s_{1}(0)=-1, \quad s_{2}(0)=0, \\
& \left|\partial_{k} s(k)\right| \leq C|\ln k|, \quad 0<k \leq k_{0} .
\end{aligned}
$$

By construction,

$$
w\left(\mathcal{F}, g_{1}\right)=2 i k, \quad w\left(\mathcal{F}, g_{3}\right)=0
$$


for any $0 \leq k<k_{0}$. As a consequence,

$$
\mathcal{F}(\rho, k)=r_{1}(k) g_{1}(\rho, k)+g_{2}(\rho, k)+r_{2}(k) g_{3}(\rho, k), \quad 0 \leq k<k_{0},
$$

with some coefficients $r_{1}(k), r_{2}(k)$ for which, by (4.8) and (4.15), we have

$$
r_{1}(0)=r_{2}(0)=0 \text {. }
$$

Computing the Wronskians $w(\mathcal{F}, \overline{\mathcal{F}})$ and $w(\mathcal{F}, \overline{\mathcal{G}})$, where $\mathcal{G}(\rho, k)=\mathcal{F}(-\rho, k)$, we get

$$
\left|s_{1}(k)\right|^{2}+\left|r_{1}(k)\right|^{2}=1, \quad r_{1}(k) \overline{s_{1}(k)}+\overline{r_{1}(k)} s_{1}(k)=0, \quad 0 \leq k<k_{0} .
$$

We can write the following Wronskian representation for $r_{1}$ :

$$
r_{1}(k)=s_{1}(k) \frac{w\left(g_{2}, f_{1}\right)}{2 i k}+s_{2}(k) \frac{w\left(g_{2}, f_{3}\right)}{2 i k}, \quad k \neq 0 .
$$

Using (4.15) and the relations

$$
\left.w\left(g_{2}, f_{3}\right)\right|_{k=0}=\left.w\left(g_{2}, f_{1}\right)\right|_{k=0}=\left.\partial_{k} w\left(g_{2}, f_{1}\right)\right|_{k=0},
$$

from (4.18) we deduce easily that $r_{1}$ is smooth for $0<k<k_{0}$ and continuous up to $k=0$, and verifies

$$
\left|\partial_{k} r_{1}(k)\right| \leq C|\ln k|, \quad 0<k<k_{0} ;
$$

in its turn, this implies that $r_{2}$ is smooth for $0<k<k_{0}$ and continuous up to $k=0$, and admits a similar estimate:

$$
\left|\partial_{k} r_{2}(k)\right| \leq C|\ln k|, \quad 0<k<k_{0} .
$$

Introduce the following odd solution of (4.2):

$$
e(\rho, k)=\mathcal{F}(-\rho, k)-\mathcal{F}(\rho, k) .
$$

By (4.14), 4.16),

$$
e=a_{1} f_{1}+f_{2}+a_{2} f_{3}, \quad a_{j}=r_{j}-s_{j}, \quad j=1,2 .
$$

From (4.15), (4.17), (4.19), 4.20) it follows that

$$
a_{1}(0)=1, \quad a_{2}(0)=0,
$$

and

$$
\left|\partial_{k} a_{j}\right| \leq C|\ln k|, \quad 0<k<k_{0}, \quad j=1,2 .
$$

Together with Lemmas 4.1 and 4.2, this implies the following result.

Lemma 4.5. (i) $e(\rho, k)=e_{0}(\rho, k)+e_{1}(\rho, k)$, where $\left.e_{0}(\rho, k)=a_{1}(k) e^{i k \rho(} \begin{array}{l}1 \\ 0\end{array}\right)+e^{-i k \rho}\left(\begin{array}{l}1 \\ 0\end{array}\right)$ and the remainder $e_{1}(\rho, k)$ admits the estimates

$$
\begin{aligned}
& \left|e_{1}(\rho, k)\right| \leq C\left(\langle\rho\rangle^{-2}+k|\ln k| e^{-k \rho}\right), \quad \rho \geq 0, \\
& \left|\partial_{k} e_{1}(\rho, k)\right| \leq C|\ln k|\left(\langle\rho\rangle^{-1}+e^{-k \rho / 2}\right), \quad \rho \geq 0, \\
& \left\|e_{1}(\cdot, k)\right\|_{L^{2}\left(\mathbb{R}_{+}\right)} \leq C, \\
& \left\|\rho e_{1}(\cdot, k)\right\|_{L^{2}\left(\mathbb{R}_{+}\right)}+\left\|\partial_{k} e_{1}(\cdot, k)\right\|_{L^{2}\left(\mathbb{R}_{+}\right)} \leq C k^{-1 / 2}|\ln k|
\end{aligned}
$$

for any $0<k \leq k_{0}$.

(ii) $\left(\rho \partial_{\rho}-k \partial_{k}\right) e(\rho, k)=e^{i k \rho}\left(\begin{array}{l}1 \\ 0\end{array}\right) k \partial_{k} a_{1}(k)+e_{2}(\rho, k)$, with $e_{2}(\rho, k)$ verifying

$$
\begin{aligned}
& \left|e_{2}(\rho, k)\right| \leq C\left(\langle\rho\rangle^{-1}+k|\ln k| e^{-k \rho / 2}\right), \quad \rho \geq 0, \\
& \left\|e_{2}(\cdot, k)\right\|_{L^{2}\left(\mathbb{R}_{+}\right)} \leq C
\end{aligned}
$$

for any $0<k \leq k_{0}$. 
For $0<\kappa \leq k_{0}$, introduce the operators $\mathbb{E}_{\kappa}: L^{2}\left(\mathbb{R}_{+}, \mathbb{C}^{2}\right) \rightarrow L^{2}\left(\mathbb{R}^{3}, \mathbb{C}^{2}\right)$,

$$
\left(\mathbb{E}_{\kappa} \Phi\right)(y)=\frac{1}{2^{3 / 2} \pi} \int_{\mathbb{R}_{+}} d k \theta_{\kappa}(k) \mathcal{E}(y, k) \Phi(k), \quad \Phi \in L^{2}\left(\mathbb{R}_{+}, \mathbb{C}^{2}\right),
$$

where $\mathcal{E}(y, k)$ is a $(2 \times 2)$ matrix given by

$$
\mathcal{E}(y, k)=\rho^{-1}\left(e(\rho, k), \sigma_{1} \overline{e(\rho, k)}\right), \quad \rho=|y|,
$$

$\theta_{\kappa}(k)=\theta\left(\kappa^{-1} k\right), \theta$ is an even $C^{\infty}$-function satisfying $\theta(k)= \begin{cases}1 & \text { if }|k| \leq 1 / 4, \\ 0 & \text { if }|k| \geq 1 / 2 .\end{cases}$

Since $e(\rho, k)$ is a solution of the equation $\widetilde{H} e=k^{2} e$, we have $H \mathbb{E}_{\kappa}=\mathbb{E}_{\kappa} k^{2} \sigma_{3}$.

By Lemma 4.5 (i), the operators $\mathbb{E}_{\kappa}$ are bounded uniformly with respect to $\kappa \leq k_{0}$. The action of the adjoint operators $\mathbb{E}_{\kappa}^{*}: L^{2}\left(\mathbb{R}^{3}, \mathbb{C}^{2}\right) \rightarrow L^{2}\left(\mathbb{R}_{+}, \mathbb{C}^{2}\right)$ is given by

$$
\left(\mathbb{E}_{\kappa}^{*} \psi\right)(k)=\frac{1}{2^{3 / 2} \pi} \theta_{\kappa}(k) \int_{\mathbb{R}^{3}} d y \mathcal{E}^{*}(y, k) \psi(y), \quad \psi \in L^{2}\left(\mathbb{R}^{3}, \mathbb{C}^{2}\right) .
$$

Clearly,

$$
\mathbb{E}_{\kappa}^{*} \sigma_{3} \zeta_{ \pm}=0
$$

for any $0<\kappa \leq k_{0}$.

The following relation is a standard consequence of the asymptotics given by Lemma 4.5 (i):

$$
\mathbb{E}_{\kappa_{2}}^{*} \sigma_{3} \mathbb{E}_{\kappa_{1}} \sigma_{3}=\theta_{\kappa_{1}}(k) \theta_{\kappa_{2}}(k)
$$

for any $0<\kappa_{1}, \kappa_{2} \leq k_{0}$.

Remark 4.6. Notice that because of the presence of the cut-off function $\theta_{\kappa}, \mathbb{E}_{\kappa}$ is bounded as an operator from $L^{2}\left(\left[0, k_{0}\right]\right)$ to $H^{m}\left(\mathbb{R}^{3}\right)$ for any $m \geq 0$, uniformly in $\kappa \leq k_{0}$.

Next we introduce quasiresonant functions $h_{\kappa}(y), 0<\kappa \leq k_{0}$, by setting

$$
h_{\kappa}=\sqrt{2} \mathbb{E}_{\kappa}\left(\begin{array}{l}
1 \\
0
\end{array}\right) \text {. }
$$

Lemma 4.7. For any $0<\kappa \leq k_{0}, h_{\kappa} \in\langle y\rangle^{-1} L^{2}\left(\mathbb{R}^{3}\right)$, and as $\kappa \rightarrow 0$ we have

$$
\begin{aligned}
& \left\|h_{\kappa}\right\|_{L^{2}\left(\mathbb{R}^{3}\right)}=O\left(\kappa^{1 / 2}\right), \quad\left\|y h_{\kappa}\right\|_{L^{2}\left(\mathbb{R}^{3}\right)}=O\left(\kappa^{-1 / 2}\right), \\
& \left\langle h_{\kappa}, \sigma_{3}\left(\xi_{0}+\xi_{1}\right)\right\rangle=4 \pi+O\left(\kappa^{1 / 2} \ln \kappa\right), \quad\left\langle h_{\kappa}, \sigma_{3}\left(\xi_{1}-\xi_{0}\right)\right\rangle=O\left(\kappa^{1 / 2} \ln \kappa\right) .
\end{aligned}
$$

Proof. Applying Lemma 4.5 (i), we decompose $h_{\kappa}$ as follows:

$$
\begin{aligned}
h_{\kappa}(y) & =h_{\kappa, 0}(y)+h_{\kappa, 1}(y)+h_{\kappa, 2}(y), \\
h_{\kappa, 0}(y) & =\frac{1}{2 \pi \rho} \kappa \hat{\theta}(\kappa \rho)\left(\begin{array}{l}
1 \\
0
\end{array}\right), \\
h_{\kappa, 1}(y) & =\frac{1}{2 \pi \rho} \int_{\mathbb{R}_{+}} d k e^{i k \rho}\left(a_{1}(k)-1\right) \theta_{\kappa}(k)\left(\begin{array}{l}
1 \\
0
\end{array}\right), \\
h_{\kappa, 2}(y) & =\frac{1}{2 \pi \rho} \int_{\mathbb{R}_{+}} d k \theta_{\kappa}(k) e_{1}(\rho, k),
\end{aligned}
$$

where $\hat{\theta}(\rho)=\int_{\mathbb{R}} e^{i k \rho} \theta(k) d k, \rho=|y|$.

Clearly, $h_{\kappa, 0} \in\langle y\rangle^{-1} L^{2}\left(\mathbb{R}^{3}\right)$, and

$$
\left\|h_{\kappa, 0}\right\|_{L^{2}\left(\mathbb{R}^{3}\right)} \leq C \kappa^{1 / 2}, \quad\left\|y h_{\kappa, 0}\right\|_{L^{2}\left(\mathbb{R}^{3}\right)} \leq C \kappa^{-1 / 2} .
$$

Consider $h_{\kappa, i}, i=1,2$. From (4.22), (4.23), (4.24) it follows that

$$
\left\|h_{\kappa, i}\right\|_{L^{2}\left(\mathbb{R}^{3}\right)} \leq C \kappa, \quad\left\|y h_{\kappa, i}\right\|_{L^{2}\left(\mathbb{R}^{3}\right)} \leq C \kappa^{1 / 2}|\ln \kappa|, \quad i=1,2,
$$


which together with (4.31) leads to the estimates

$$
\left\|h_{\kappa}\right\|_{L^{2}\left(\mathbb{R}^{3}\right)} \leq C \kappa^{1 / 2}, \quad\left\|y h_{\kappa}\right\|_{L^{2}\left(\mathbb{R}^{3}\right)} \leq C \kappa^{-1 / 2} .
$$

Next we compute $\left\langle h_{\kappa}, \sigma_{3}\left(\xi_{1} \pm \xi_{0}\right)\right\rangle$. By (4.31) and (4.32), as $\kappa \rightarrow 0$ we have

$$
\begin{aligned}
\left\langle h_{\kappa}, \sigma_{3}\left(\xi_{1} \pm \xi_{0}\right)\right\rangle & =\left\langle h_{\kappa, 0}, \sigma_{3}\left(\xi_{1} \pm \xi_{0}\right)\right\rangle+O\left(\kappa^{1 / 2} \ln \kappa\right), \\
\left\langle h_{\kappa, 0}, \sigma_{3}\left(\xi_{1}-\xi_{0}\right)\right\rangle & =O(\kappa), \\
\left\langle h_{\kappa, 0}, \sigma_{3}\left(\xi_{1}+\xi_{0}\right)\right\rangle & =2 \kappa \int_{\mathbb{R}} d \rho \hat{\theta}(\kappa \rho)+O(\kappa)=4 \pi+O(\kappa),
\end{aligned}
$$

which gives (4.29).

4.3. Proof of Proposition 3.1. We start with deriving some coercivity bounds for the operator $H$.

Lemma 4.8. There exists $\kappa_{0}, 0<\kappa_{0} \leq k_{0}$, and $C>0$ such that

$$
\left\langle H f, \sigma_{3} f\right\rangle \geq C \kappa\|\nabla f\|_{L^{2}\left(\mathbb{R}^{3}\right)}^{2}
$$

for any $0<\kappa \leq \kappa_{0}$ and any $f \in \dot{H}_{\mathrm{rad}}^{1}\left(\mathbb{R}^{3}, \mathbb{C}^{2}\right)$ verifying

$$
\left\langle f, \sigma_{3} \zeta_{-}\right\rangle=\left\langle f, \sigma_{3} \zeta_{+}\right\rangle=\left\langle f, \sigma_{3} h_{\kappa}\right\rangle=\left\langle f, \sigma_{3} \sigma_{1} \bar{h}_{\kappa}\right\rangle=0 .
$$

Remark 4.9. Notice that, since $\zeta_{ \pm}, h_{\kappa} \in\langle y\rangle^{-1} L^{2}\left(\mathbb{R}^{3}\right)$, the scalar products involved in (4.36) are well defined for any $f \in \dot{H}^{1}$.

Proof. The proof of Lemma 4.8 is based on the following result, which is due to Duyckaerts and Merle.

Lemma 4.10. There exists $c_{0}>0$ such that

$$
\left\langle H f, \sigma_{3} f\right\rangle \geq c_{0}\|\nabla f\|_{L^{2}\left(\mathbb{R}^{3}\right)}^{2}
$$

for any $f \in \dot{H}_{\text {rad }}^{1}\left(\mathbb{R}^{3}, \mathbb{C}^{2}\right)$ verifying

$$
\left\langle f, \sigma_{3} \zeta_{-}\right\rangle=\left\langle f, \sigma_{3} \zeta_{+}\right\rangle=\left\langle f, \Delta \xi_{0}\right\rangle=\left\langle f, \Delta \xi_{1}\right\rangle=0,
$$

see [5] for the proof.

Let $f \in \dot{H}_{\text {rad }}^{1}$ be such that (4.36) is fulfilled. We can write $f$ as

$$
f=\alpha_{0} \xi_{0}+\alpha_{1} \xi_{1}+g,
$$

where

$$
\alpha_{j}=-\frac{\left\langle f, \Delta \xi_{j}\right\rangle}{\left\|\nabla \xi_{j}\right\|_{L^{2}\left(\mathbb{R}^{3}\right)}^{2}}, \quad j=0,1,
$$

and $g \in \dot{H}_{\text {rad }}^{1}$ verifies

$$
\left\langle g, \sigma_{3} \zeta_{-}\right\rangle=\left\langle g, \sigma_{3} \zeta_{+}\right\rangle=\left\langle g, \Delta \xi_{0}\right\rangle=\left\langle g, \Delta \xi_{1}\right\rangle=0 .
$$

Therefore, by Lemma 4.10 .

$$
\left\langle H f, \sigma_{3} f\right\rangle=\left\langle H g, \sigma_{3} g\right\rangle \geq c_{0}\|\nabla g\|_{L^{2}\left(\mathbb{R}^{3}\right)}^{2} .
$$

Furthermore, since $f$ satisfies (4.36), we have

$$
A(\kappa)\left(\begin{array}{c}
\alpha_{0} \\
\alpha_{1}
\end{array}\right)=\left(\begin{array}{c}
\left\langle g, \sigma_{3} h_{\kappa}\right\rangle \\
\left\langle g, \sigma_{3} \sigma_{1} \bar{h}_{\kappa}\right\rangle
\end{array}\right),
$$

where

$$
A(\kappa)=-\left(\begin{array}{cc}
\left\langle\xi_{0}, \sigma_{3} h_{\kappa}\right\rangle & \left\langle\xi_{1}, \sigma_{3} h_{\kappa}\right\rangle \\
\left\langle h_{\kappa}, \sigma_{3} \xi_{0}\right\rangle & -\left\langle h_{\kappa}, \sigma_{3} \xi_{1}\right\rangle
\end{array}\right) .
$$


By (4.29),

$$
A(\kappa)=-2 \pi\left(\begin{array}{cc}
1 & 1 \\
1 & -1
\end{array}\right)+O\left(\kappa^{1 / 2} \ln \kappa\right), \quad \kappa \rightarrow 0 .
$$

Therefore, if $\kappa$ is sufficiently small, then

$$
\left|\alpha_{0}\right|+\left|\alpha_{1}\right| \leq C\|\nabla g\|_{L^{2}\left(\mathbb{R}^{3}\right)}\left\|\langle y\rangle h_{\kappa}\right\|_{L^{2}\left(\mathbb{R}^{3}\right)} \leq C \kappa^{-1 / 2}\|\nabla g\|_{L^{2}\left(\mathbb{R}^{3}\right)} .
$$

As a consequence,

$$
\|\nabla f\|_{L^{2}\left(\mathbb{R}^{3}\right)} \leq C \kappa^{-1 / 2}\|\nabla g\|_{L^{2}\left(\mathbb{R}^{3}\right)} .
$$

Combining this inequality with (4.37), we get (4.35).

Lemma 4.11. There exists $\kappa_{1}, 0<\kappa_{1} \leq k_{0}$, and $C>0$ such that for any $0<\kappa \leq \kappa_{1}$ we have

$$
\|f\|_{H^{1}\left(\mathbb{R}^{3}\right)} \leq \frac{C}{\kappa}\|\nabla f\|_{L^{2}\left(\mathbb{R}^{3}\right)}
$$

for all $f \in H_{\mathrm{rad}}^{1}\left(\mathbb{R}^{3}\right)$ with $\mathbb{E}_{\kappa}^{*} f=0$.

Proof. By (4.22), (4.23), and Lemma 4.5 (i), $\mathbb{E}_{\kappa}^{*} f$ can be written as

$$
\left(\mathbb{E}_{\kappa}^{*} f\right)(k)=\Phi_{0}(k)+\Phi_{r}(k),
$$

where

$$
\Phi_{0}(k)=\frac{1}{2^{3 / 2} \pi} \theta_{\kappa}(k) \check{f}(k),
$$

$\breve{f}(k)=2 \int_{\mathbb{R}^{3}} d y \frac{\cos k|y|}{|y|} f(y)$, and the remainder $\Phi_{r}$ satisfies

$$
\left\|\Phi_{r}\right\|_{L^{2}\left(\mathbb{R}_{+}\right)} \leq C \kappa^{1 / 2}\|f\|_{L^{2}\left(\mathbb{R}^{3}\right)} .
$$

Therefore, $\mathbb{E}_{\kappa}^{*} f=0$ implies

$$
\|\breve{f}\|_{L^{2}(0, \kappa / 4)} \leq C \kappa^{1 / 2}\|f\|_{L^{2}\left(\mathbb{R}^{3}\right)} .
$$

Notice also that for any $f \in H_{\text {rad }}^{1}$ and any $0<\kappa \leq 1$ we have

$$
\|f\|_{H^{1}\left(\mathbb{R}^{3}\right)} \leq C\left(\|\check{f}\|_{L^{2}(0, \kappa / 4)}+\kappa^{-1}\|\nabla f\|_{L^{2}\left(\mathbb{R}^{3}\right)}\right) .
$$

Combining this inequality with (4.38), we get

$$
\|f\|_{H^{1}\left(\mathbb{R}^{3}\right)} \leq \frac{C}{\kappa}\|\nabla f\|_{L^{2}\left(\mathbb{R}^{3}\right)}
$$

provided $\kappa$ is sufficiently small.

Finally, we combine Lemmas 4.8 and 4.11 to derive the following result, which will be in the heart of the proof of Proposition 3.1

Lemma 4.12. There exists $\kappa_{2}, 0<\kappa_{2} \leq k_{0}$, and $C>0$ such that for any $0<\kappa \leq \kappa_{2}$ we have

$$
\left\langle H f, \sigma_{3} f\right\rangle \geq C \kappa^{3}\|f\|_{H^{1}}^{2}-\frac{\kappa}{C}\left\|\mathbb{E}_{\kappa}^{*} \sigma_{3} f\right\|_{L^{2}\left(\mathbb{R}_{+}\right)}^{2}
$$

for any $f \in H_{\mathrm{rad}}^{1}\left(\mathbb{R}^{3}, \mathbb{C}^{2}\right)$ with $\left\langle f, \sigma_{3} \zeta_{ \pm}\right\rangle=0$.

Proof. Write $f=f_{1}+f_{2}$, where $f_{1}=\mathbb{E}_{\kappa} \sigma_{3} \mathbb{E}_{\kappa}^{*} \sigma_{3} f$ and $f_{2}=f-f_{1}$. Clearly,

$$
\left\|f_{1}\right\|_{H^{1}\left(\mathbb{R}^{3}\right)} \leq C\left\|\mathbb{E}_{\kappa}^{*} \sigma_{3} f\right\|_{L^{2}\left(\mathbb{R}_{+}\right)}, \quad\left\|H f_{1}\right\|_{L^{2}\left(\mathbb{R}^{3}\right)} \leq C \kappa^{2}\left\|\mathbb{E}_{\kappa}^{*} \sigma_{3} f\right\|_{L^{2}\left(\mathbb{R}_{+}\right)},
$$

for any $0<\kappa \leq k_{0}$.

Consider $f_{2}$. From (4.26) and (4.27) it follows that for any $\kappa^{\prime} \leq \kappa / 2$,

- $\left\langle f_{2}, \sigma_{3} \zeta_{ \pm}\right\rangle=0$

- $\mathbb{E}_{\kappa^{\prime}}^{*} \sigma_{3} f_{2}=0$;

- $\left\langle f_{2}, \sigma_{3} h_{\kappa^{\prime}}\right\rangle=\left\langle f_{2}, \sigma_{3} \sigma_{1} \bar{h}_{\kappa^{\prime}}\right\rangle=0$. 
Hence, by Lemmas 4.8 and 4.11 we have

$$
\left\langle H f_{2}, \sigma_{3} f_{2}\right\rangle \geq C \kappa^{3}\left\|f_{2}\right\|_{H^{1}\left(\mathbb{R}^{3}\right)}^{2},
$$

provided $\kappa$ is sufficiently small.

Combining (4.40) and (4.41), we arrive at (4.39).

Now we are in a position to prove Proposition 3.1. Consider the equation

$$
\begin{aligned}
& i \psi_{\tau}=P \mathcal{H}(\tau) P \psi, \\
& \left.\psi\right|_{\tau=s}=f,
\end{aligned}
$$

where

$$
\mathcal{H}(\tau)=H+\tau^{-1} l, \quad l=\alpha_{1} \sigma_{3}-i \nu_{1}\left(\frac{1}{2}+y \cdot \nabla\right),
$$

$\alpha_{1}, \nu_{1} \in \mathbb{R}, s>0$, and $f \in \mathcal{S}\left(\mathbb{R}^{3}\right)$ satisfies $\left\langle f, \sigma_{3} \zeta_{ \pm}\right\rangle=0$.

Fix $\kappa$ such that $0<\kappa \leq \kappa_{2}$ and consider the functional $G_{1}(\tau)=\left\langle H \psi, \sigma_{3} \psi\right\rangle+$ $c_{0}\left\|\mathbb{E}_{\kappa}^{*} \sigma_{3} \psi\right\|_{L^{2}\left(\mathbb{R}_{+}\right)}^{2}$. Clearly,

$$
G_{1}(\tau) \leq C\|\psi(\tau)\|_{H^{1}\left(\mathbb{R}^{3}\right)}^{2} .
$$

Moreover, since $\left\langle\psi(\tau), \sigma_{3} \zeta_{ \pm}\right\rangle=0$, choosing $c_{0}$ sufficiently large, we get

$$
G_{1}(\tau) \geq c_{1}\|\psi(\tau)\|_{H^{1}\left(\mathbb{R}^{3}\right)}^{2} .
$$

Next we compute the derivative $\frac{d}{d \tau} G_{1}$. We have

which implies

$$
i \frac{d}{d \tau}\left\langle H \psi, \sigma_{3} \psi\right\rangle=\frac{2 i}{\tau} \operatorname{Im}\left\langle l \psi, \sigma_{3} H \psi\right\rangle
$$

$$
\left|\frac{d}{d \tau}\left\langle H \psi, \sigma_{3} \psi\right\rangle\right| \leq \frac{C}{\tau}\left(\left|\alpha_{1}\right|+\left|\nu_{1}\right|\right)\|\nabla \psi(\tau)\|_{L^{2}\left(\mathbb{R}^{3}\right)}^{2} .
$$

Now, we address $\left\|\mathbb{E}_{\kappa}^{*} \sigma_{3} \psi\right\|_{L^{2}\left(\mathbb{R}_{+}\right)}^{2}$. Denote $\Phi(\tau)=\mathbb{E}_{\kappa}^{*} \sigma_{3} \psi(\tau)$. Then $\Phi(k, \tau)$ solves

$$
i \Phi_{\tau}=k^{2} \sigma_{3} \Phi+\frac{1}{\tau} Y
$$

where

$$
Y=\mathbb{E}_{\kappa}^{*} \sigma_{3} l \psi
$$

Integrating by parts and applying Lemma 4.5 (ii), we can rewrite $Y$ in the form

$$
Y(k, \tau)=Y_{0}(k, \tau)+Y_{1}(k, \tau),
$$

where

$$
Y_{0}(k, \tau)=i \nu_{1} k \partial_{k} \Phi(k, \tau)
$$

and $Y_{1}(k, \tau)$ admits the estimate

$$
\left\|Y_{1}(\tau)\right\|_{L^{2}\left(\mathbb{R}_{+}\right)} \leq C\left(\left|\alpha_{1}\right|+\left|\nu_{1}\right|\right)\|\psi(\tau)\|_{L^{2}\left(\mathbb{R}^{3}\right)} .
$$

Therefore, (4.46) gives

$$
\left|\frac{d}{d \tau}\|\Phi(\tau)\|_{L^{2}\left(\mathbb{R}_{+}\right)}^{2}\right| \leq \frac{C}{\tau}\left(\left|\alpha_{1}\right|+\left|\nu_{1}\right|\right)\|\psi(\tau)\|_{L^{2}\left(\mathbb{R}^{3}\right)}^{2} .
$$

Combining this inequality with (4.46) and taking (4.44) into account, we get

$$
\left|\frac{d}{d \tau} G_{1}(\tau)\right| \leq \frac{C}{\tau}\left(\left|\alpha_{1}\right|+\left|\nu_{1}\right|\right)\|\psi(\tau)\|_{H^{1}\left(\mathbb{R}^{3}\right)}^{2} \leq \frac{C}{\tau}\left(\left|\alpha_{1}\right|+\left|\nu_{1}\right|\right) G_{1}(\tau) .
$$

Integration yields

$$
G_{1}(\tau) \leq C\left(\frac{s}{\tau}\right)^{C\left(\left|\alpha_{1}\right|+\left|\nu_{1}\right|\right)} G_{1}(s), \quad 0<\tau \leq s
$$


which, by (4.43) and (4.44), leads to the bound

$$
\|U(\tau, s) f\|_{H^{1}\left(\mathbb{R}^{3}\right)} \leq C\left(\frac{s}{\tau}\right)^{C\left(\left|\alpha_{1}\right|+\left|\nu_{1}\right|\right)}\|f\|_{H^{1}\left(\mathbb{R}^{3}\right)}
$$

for any $0<\tau \leq s$ and any $f \in H_{\mathrm{rad}}^{1}$.

To control higher regularity, consider the functional $G_{2}(\tau)=\left\langle H^{2} \psi, \sigma_{3} H \psi\right\rangle+c_{2} G_{1}(\tau)$. We have

$$
C^{-1}\|\psi\|_{H^{3}\left(\mathbb{R}^{3}\right)}^{2} \leq G_{2} \leq C\|\psi\|_{H^{3}\left(\mathbb{R}^{3}\right)}^{2},
$$

provided $c_{2}$ is chosen sufficiently large.

Computing the derivative $\frac{d}{d \tau}\left\langle H^{2} \psi(\tau), \sigma_{3} H \psi(\tau)\right\rangle$ and using (4.47), we get

$$
\left|\frac{d}{d \tau} G_{2}(\tau)\right| \leq \frac{C}{\tau}\left(\left|\alpha_{1}\right|+\left|\nu_{1}\right|\right)\|\psi(\tau)\|_{H^{3}\left(\mathbb{R}^{3}\right)}^{2} \leq \frac{C}{\tau}\left(\left|\alpha_{1}\right|+\left|\nu_{1}\right|\right) G_{2}(\tau) .
$$

which implies

$$
\|U(\tau, s) f\|_{H^{3}\left(\mathbb{R}^{3}\right)} \leq C\left(\frac{s}{\tau}\right)^{C\left(\left|\alpha_{1}\right|+\left|\nu_{1}\right|\right)}\|f\|_{H^{3}\left(\mathbb{R}^{3}\right)}
$$

for any $0<\tau \leq s$.

The $H^{2}$ bound stated in Proposition 3.1 follows from (4.48) and (4.50) by interpolation.

\section{REFERENCES}

[1] V. S. Buslaev and G. S. Perelman, Scattering for the nonlinear Schrödinger equation: states close to a soliton, Algebra i Analiz 4 (1992), no. 6, 63-102; English transl., St. Petersburg Math. J. 4 (1993), 1111-1142. MR1199635(94b:35256)

[2] Th. Cazenave, Semilinear Schrödinger equations, Courant Lecture Notes in Math., vol. 10, Courant Inst. Math. Sci., New York; Amer. Math. Soc., Providence, RI, 2003. MR2002047 (2004j:35266)

[3] Th. Cazenave and F. B. Weissler, The Cauchy problem for the critical nonlinear Schrödinger equation in $H^{s}$, Nonlinear Anal. 14 (1990), no. 10, 807-836. MR.1055532 (91j:35252)

[4] R. Donninger and J. Krieger, Nonscattering solutions and blow up at infinity for the critical wave equation, Preprint, 2012; arXiv:1201.3258.

[5] Th. Duyckaerts and F. Merle, Dynamic of threshold solutions for energy-critical NLS, Geom. Funct. Anal. 18 (2009), no. 6, 1787-1840. MR2491692 (2010e:35258)

[6] C. Kenig and F. Merle, Global well-posedness, scattering and blow-up for the energy-critical, focusing, non-linear Schrödinger equation in the radial case, Invent. Math. 166 (2006), no. 3, 645-675. MR2257393(2007g:35232)

[7] J. Krieger and W. Schlag, Stable manifolds for all monic supercritical focusing nonlinear Schrödinger equations in one dimension, J. Amer. Math. Soc. 19 (2006), no. 4, 815-920. MR2219305 (2007b:35301)

[8] J. Krieger, W. Schlag, and D. Tataru, Slow blow-up solutions for the $H^{1}\left(\mathbb{R}^{3}\right)$ critical focusing semilinear wave equation, Duke Math. J. 147 (2009), no. 1, 1-53. MR2494455 (2010h:58045)

[9] G. Perelman, Blow up dynamics for equivariant critical Schrödinger maps, Preprint, 2012.

Université Paris-Est Créteil, Créteil Cedex, France

E-mail address: cecilia.ortoleva@math.cnrs.fr

Université Paris-Est Créteil, Créteil Cedex, France

E-mail address: galina.perelman@u-pec.fr

Received 2/OCT/2012

Originally published in English 